\title{
Maternidad, infancia y drogas: implicaciones clínicas
}

\author{
Helena Calvo Botella \\ Centro de Día PATIM. Psicóloga. Master en Prevención y Tratamiento de Conductas Adictivas. \\ Enviar correspondencia a: \\ Helena Calvo Botella. PATIM Plaza Tetuán 9, 3 CP 12001 Castellón. E-mail: hcalvo@ono.com
}

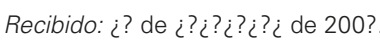
Aceptado: $i$ ? de $i ? i ? i$ ? de 200 ?

\section{RESUMEN}

Objetivos: Revisar la relación entre la exposición prenatal a drogas y el pronóstico fetal, neonatal e infantil. Material y métodos: se estudia el estado actual de la investigación relativa a la exposición prenatal a sustancias psicoactivas, mediante una extensa revisión de Medline y de las investigaciones de organismos norteamericanas y europeos durante los últimos años. Se presta especial atención a las dificultades metodológicas derivadas del objeto de estudio y a otros factores tales como la relación materno-infantil y las características de la mujer adicta. Resultados: la exposición prenatal a drogas se asocial a un amplio rango de complicaciones obstétricas y alteraciones neuroconductuales infantiles. Con la excepción del síndrome alcohólico fetal y otros efectos fetales ligados al alcohol, estas alteraciones suelen encontrarse en dominios cognitivos específicos y pueden estar moduladas por factores psicosociales y ambientales. Conclusiones: los niños expuestos prenatalmente a drogas pueden ser considerados un caso especial de infancia en riesgo. Es necesario investigar el impacto real de la exposición prenatal a sustancias psicoactivas y la relevancia de otros factores en su pronóstico a medio y largo plazo. Asimismo, es necesario implicar y formar a diferentes profesionales, tales como obstetras, ginecólogos y pediatras, y coordinar la intervención de las diferentes instituciones, para mejorar la atención prestada a la mujer drogodependiente y a sus hijos.

Palabras clave: mujer, droga, teratógeno, embarazo de alto riesgo, manifestaciones neuroconductuales.

\section{ABSTRACT}

Objective: To review the relationship between prenatal drug exposure and foetal, neonatal and infant outcome. Material and methods: We study the current status of findings on prenatal exposure to psychoactive substances by means of an extensive review of Medline and North American and European research in the last few years. We pay special attention to the methodological complications arising from the objective of the study and to other factors such as the mother-infant relationship and the characteristics of drug dependent woman. Results: Prenatal drug exposure can be associated with a wide range of obstetrical complications and neurobehavioral disturbances in infants. With the exception of foetal alcohol syndrome and other foetal effects linked to alcohol, these alterations tend to be found in specific cognitive domains and can be modulated by psychosocial and environmental factors. Conclusion: infants prenatally exposed to drugs can be considered to be a special case of infancy at risk. It is necessary to investigate the real impact of prenatal drug exposure and the significance of other factors in the medium and long-term. In addition, it is essential to involve and train the various professionals, such as obstetricians, gynaecologists and paediatricians, and to coordinate the interventions of the different institutions, in order to improve the attention given to drug dependent women and their children.

Key words: woman, drug, teratogen, high-risk pregnancy, neurobehavioral manifestations.

\section{INTRODUCCIÓN}

E abuso de sustancias psicoactivas y las complicaciones sociosanitarias derivadas del mismo constituyen un problema de importante magnitud. En este sentido, la acepción cotidiana de la palabra "abuso", sobre todo cuando se trata de sustancias de curso legal, suele referirse, de modo implícito, al uso de dicha sustancia en cantidades excesivas o por encima de lo que se considera "habitual". No obstante, la cantidad consumida no es un criterio unívoco de abuso, desde el momento que determinados colectivos o poblaciones, por circunstancias personales, se ven abocadas a un daño o perjuicio potencial en cantidades que en otras situaciones podrían considerarse "normativas". Uno de estos colectivos es el de la mujer embarazada, en la que el consumo de dro- 
gas es susceptible de afectar al normal desarrollo del feto. Por ello revisaremos en estas líneas los datos existentes acerca de las consecuencias derivadas del consumo de drogas a lo largo del periodo de gestación, prestando asimismo atención a otros factores que puedan mediatizar dichas consecuencias.

\section{TERATOGÉNESIS Y EXPOSICIÓN PRENATAL A SUSTANCIAS PSICOACTIVAS}

El concepto más "clásico" de teratogénesis hace referencia a la capacidad de un determinado agente de inducir malformaciones de carácter estructural en un organismo en desarrollo, idea que enlaza con la etimología del término (del griego téras. tératos, monstruo). No obstante, progresivamente esta concepción se ha ido ampliando ${ }^{1,2}$, definiéndose como agente teratógeno aquel cuya exposición al mismo durante el desarrollo del organismo es capaz de inducir en éste alteraciones morfológicas, bioquímicas o funciona$l_{e s}{ }^{3}$. Esto ha llevado a la consideración de que determinados agentes podrían actuar como teratógenos conductuales o funcionales ${ }^{2,4}$, alterando el sistema nervioso en ausencia de malformaciones evidentes y manifestándose mediante la presencia de alteraciones neuroconductuales diversas a lo largo del desarrollo infantil ${ }^{5}$. Otro término relativo a estos agentes sería el de neuroteratógeno².

La clasificación de un determinado agente como teratógeno debe hacer referencia no sólo a las características de dicho agente, sino a la dosis, la especie sobre la que se estudian sus efectos, y el periodo de gestación que concurre con la exposición ${ }^{6}$. Este último aspecto supone la existencia de periodos críticos, de amplitud variable, para cada tipo de agente $y$, especialmente, para cada tipo de malformación y/o efecto $^{6}$. En referencia a la dosis, se observa un fenómeno de dosis-respuesta por encima de un determinado umbral, por debajo del cual no se observarían efectos teratógenos y por encima del cual se produciría un incremento en la frecuencia y severidad de la respuesta en relación al incremento en la dosis ${ }^{6,7}$. No obstante, este umbral es a menudo difícil de establecer. Quizá el caso más representativo es el del síndrome alcohólico fetal, un cuadro de embriofetopatía debida al consumo de alcohol durante el embarazo y para el que no existe, tras varias décadas de investigación, una dosis de consumo "segura"1,8. Este fenómeno dosis-dependiente puede estudiarse en modelos animales permitiendo un riguroso control de las condiciones bajo las que se produce la exposición al agente; sin embargo, existen diferencias interespecíficas en metabolismo, vía de administración, patrones temporales en la maduración del desarrollo fetal, susceptibilidad genética a cada agente y estructura y función de la placenta ${ }^{1,6,9-11}$, que condicionan el grado en que los resultados obtenidos sobre modelos animales pueden considerarse extensivos a la gestación humana y obligan a diseños metodológicos precisos. En este sentido, la investigación sobre teratogénesis en humanos debe tener en cuenta una serie de criterios adicionales ${ }^{6}$.

- Constatación a partir de estudios epidemiológicos de un incremento en la incidencia de un espectro particular de malformaciones congénitas en poblaciones humanas expuestas al agente.

- Los rasgos seculares deben demostrar una relación entre la incidencia de una malformación particular y los cambios en la exposición al agente en la población.

- Un modelo animal mimetiza las malformaciones humanas a niveles clínicamente comparables.

- El efecto teratogéno aumenta con la dosis.

- Los mecanismos de teratogénesis son comprendidos y/o son biológicamente plausibles.

Para la teratogénesis clásica, la presencia de malformaciones estructurales tiene como periodo crítico el primer trimestre de gestación, en el que se produce la organogénesis. No obstante, en el caso de la teratogénesis "funcional", los periodos críticos hacen referencia a las diversas etapas de desarrollo cerebral ${ }^{12}$ y a la capacidad del agente teratógeno de influir en estos procesos. Las drogas de abuso poseen potencialmente dicha capacidad, tanto a través de efectos directos como indirectos. Un ejemplo de efectos indirectos serían los fenómenos de hipoxia e isquemia secundarios al consumo materno de cocaína o nicotina ${ }^{2,4,9}$, mientras que los efectos directos vendrían dados por los efectos de la sustancia o sus metabolitos en los sistemas de neurotransmisión del cerebro en desarrollo ${ }^{4,5}$, cuyas funciones y características difieren de las del cerebro adulto ${ }^{2,13}$. Dado que la mayor parte de drogas de abuso tiene un paso comprobado a través de la placenta ${ }^{14}$, la sobreestimulación de los sistemas de neurotransmisión del feto puede producir anomalías en los patrones de proliferación, diferenciación y migración neuronal, que son parcialmente controlados por estos sistemas de neurotransmisión²,13,15, así como alteraciones persistentes en la responsividad de la célula y alteraciones secundarias de carácter compensatorio que contribuyan a déficits conductuales posteriores ${ }^{2,15}$.

Debe señalarse que la teratogénesis funcional, del mismo modo que la teratogénesis clásica, debe ser explicada a varios niveles: cognitivo (qué déficit se produce), sistémico (qué estructuras cerebrales y sistemas neuroquímicos subyacen a dichos déficits), temporal (en qué periodo se produce la vulnerabilidad 
para el agente) y fármaco-fisiológico (cuál es el mecanismo de acción del mismo) ${ }^{4}$.Especialmente interesante a este respecto es la propuesta de Lester et al ${ }^{5}(1996)$, para quienes la afectación neuroconductual derivada de la exposición prenatal a drogas, lejos de presentarse en forma de alteraciones generalizadas, se produce en dominios cognitivos específicos (Ver Figura 1).

Existen sin embargo numerosas complicaciones metodológicas asociadas a la investigación sobre la exposición prenatal a sustancias psicoactivas. Una de las más relevantes hacen referencia a la obtención de información sobre las condiciones y características de la exposición prenatal a la sustancia psicoactiva, así como la categorización y manejo de dichos datos ${ }^{7,16}$. Los modelos animales, a pesar del elevado grado de control experimental que suponen, tienen dos importantes desventajas: en primer lugar, el grado de comparabilidad específica puede ser elevado una vez establecidos los consecuentes controles experimentales, pero la equivalencia nunca será exacta, y en segundo lugar, permiten obtener información sobre los efectos neurobiológicos directos de la sustancia y su potencial teratógeno, pero no pueden evaluar el impacto de otros factores asociados al abuso de sustancias ${ }^{9}$, o bien no permiten estudiar adecuadamente el compromiso de funciones cognitivas superiores ${ }^{1}$.

En referencia a estos aspectos, en los últimos años se está haciendo un importante esfuerzo a la hora de realizar estudios de corte preferentemente prospectivo en muestras humanas. Existe en este ámbito una notable polémica acerca de las ventajas o desventajas del empleo de biomarcadores como indicadores del consumo de drogas por la gestante frente al autoinforme materno. Los biomarcadores ofrecen una serie de ventajas e inconvenientes (invasividad, estabilidad de la muestra, soporte técnico necesario para su análisis) que son evaluadas a la hora de su elección, así como un grado relativo de seguridad acerca de si ha existido o no exposición, en función del tipo y características del biomarcador utilizado y el momento en que dicha exposición se produjo ${ }^{17}$. El análisis cuantitativo de la concentración de metabolitos en el biomarcador (meconio o cabello) se ha utilizado para establecer el grado relativo de exposición a la sustancia durante el embarazo y su correlación con determinados parámetros ${ }^{18}$. Sin embargo, la capacidad del biomarcador para ofrecer información precisa sobre los patrones de consumo a lo largo del embarazo es limitada y en algunos casos nula. En este sentido se ha señalado que la mejor fuente de información sobre el tipo, cantidad, frecuencia y duración del consumo de drogas a lo largo del embarazo es el autoinforme materno ${ }^{7}$. Aunque la negación del consumo por miedo a la estigmatización y los sesgos de memoria son algunos de los argumentos más frecuentemente utilizados por los defensores del uso de biomarcadores, lo cierto es que existen estudios en los que el autoinforme ha sido más fiable que el biomarcador ${ }^{19}$, por lo que el uso de estrategias orientadas a minimizar la negación y contextualizar las características del consumo es común al ámbito de la investigación y la intervención ${ }^{7,20}$. La combinación de biomarcador más autoinforme es posiblemente la opción más acertada, aunque es importante señalar que dado que la inconsistencia en los resultados obtenidos a partir de ambos métodos puede obedecer a múltiples causas $^{21}$, no es infrecuente que la asignación al grupo de

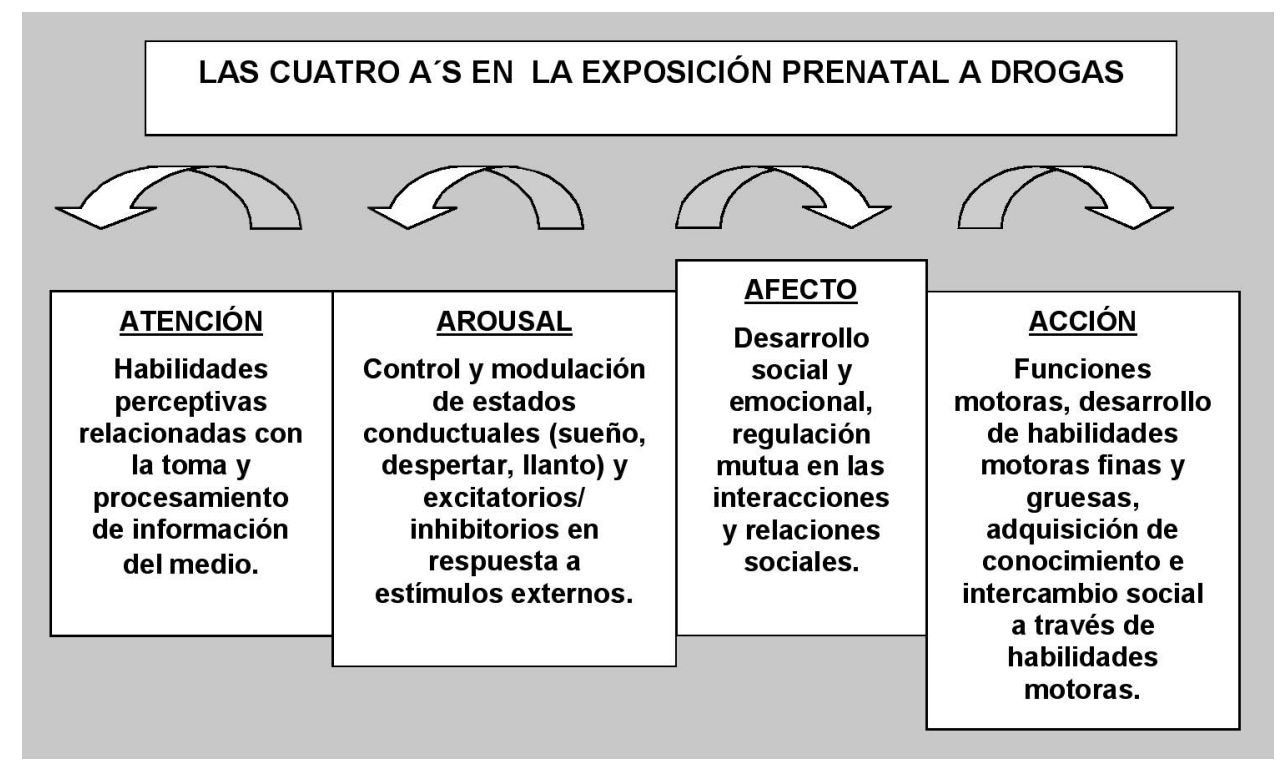

FIGURA 1

Las cuatro A’s de la exposición prenatal a drogas según Lester, LaGasse, Freier y Brunner, 1996 
neonatos expuestos se haga en base al positivo en al menos una de las pruebas.

Otra problemática frecuente en este tipo de estudios es la presencia de politoxicomanía materna, o el policonsumo de sustancias cuando no existe adicción. La politoxicomanía y el policonsumo son frecuentes en casi todas las muestras ${ }^{16,22,23}$ por lo que a menudo aislar el efecto directo de una sustancia en un determinado parámetro sólo es -parcialmente- viable a partir de estrategias estadísticas. Asimismo, la politoxicomanía puede producir exposiciones sucesivas o simultáneas a varias sustancias psicoactivas a lo largo del desarrollo fetal, lo que puede enmascarar los efectos de una droga con los de otra ${ }^{24}$ o dar como resultado efectos diferentes en virtud de la sinergia que puede darse entre dichas sustancias ${ }^{5,7}$. Una vez más, resulta complejo saber hasta qué punto es posible y/o conveniente (dada la elevada frecuencia de policonsumos) considerar el efecto de cada droga de forma independiente.

Por otro lado, la toxicidad directa de una sustancia es un factor relevante, pero no el único, a la hora de explicar el pronóstico a corto, medio y largo plazo del niño prenatalmente expuesto a drogas. El consumo de sustancias psicoactivas por parte de la gestante puede asociarse a complicaciones obstétricas diversas derivadas tanto de los efectos de la sustancia misma, como del estilo de vida en el que se ve inmersa la mujer drogodependiente (ver Figura 2) 14,16,22,24, lo que amplía el riesgo que el consumo de una droga tiene sobre el feto más allá de potencial teratógeno -estructural o funcional- de la misma. Dado que muchas de estas complicaciones pueden comprometer la evolución de la gestación y afectar al desa- rrollo del niño durante la primera infancia, los efectos de este tipo de variables (prematuridad, desnutrición, bebés de bajo peso, procesos infecciosos) deben ser considerados. Un ejemplo de ello es la relación que se da entre cocaína, prematuridad y bebés de bajo peso ${ }^{5,25}$. De manera independiente estas variables pueden comprometer el desarrollo del niño, por lo que, en teoría, sería conveniente explorar los efectos que la cocaína tiene sobre el neonato en embarazos llevados a término y en ausencia de déficits de crecimiento. Sin embargo, lo cierto es que según la literatura, la cocaína se asocia a prematuridad y déficits en el crecimiento intrauterino, por lo que desligar estos aspectos puede resultar difícil. La cuestión a este respecto podría resumirse, básicamente, en si los diversos efectos de la cocaína sobre el neonato están mediados por la prematuridad, son independientes, o se potencian mutuamente.

Finalmente, el grado de sensibilidad de los instrumentos de evaluación, y las condiciones y momentos en los que se produce la misma, pueden afectar a los resultados obtenidos. Atendiendo al hecho de que, tal y como se ha señalado anteriormente, en el mayor número de casos los efectos provocados por la exposición prenatal a drogas harían referencia a dominios cognitivos concretos, se ha señalado la necesidad de desarrollar instrumentos de evaluación más específicos que los habitualmente empleados $5,25,26$, caso de los índices de desarrollo mental y psicomotor de las Escalas Bayley de desarrollo infantil. A pesar de que han sido muy utilizadas en el estudio de los efectos de diversos teratógenos, las Escalas Bayley evalúan aspectos excesivamente generales y han demostrado tener poca validez predictiva en la competencia cogni-

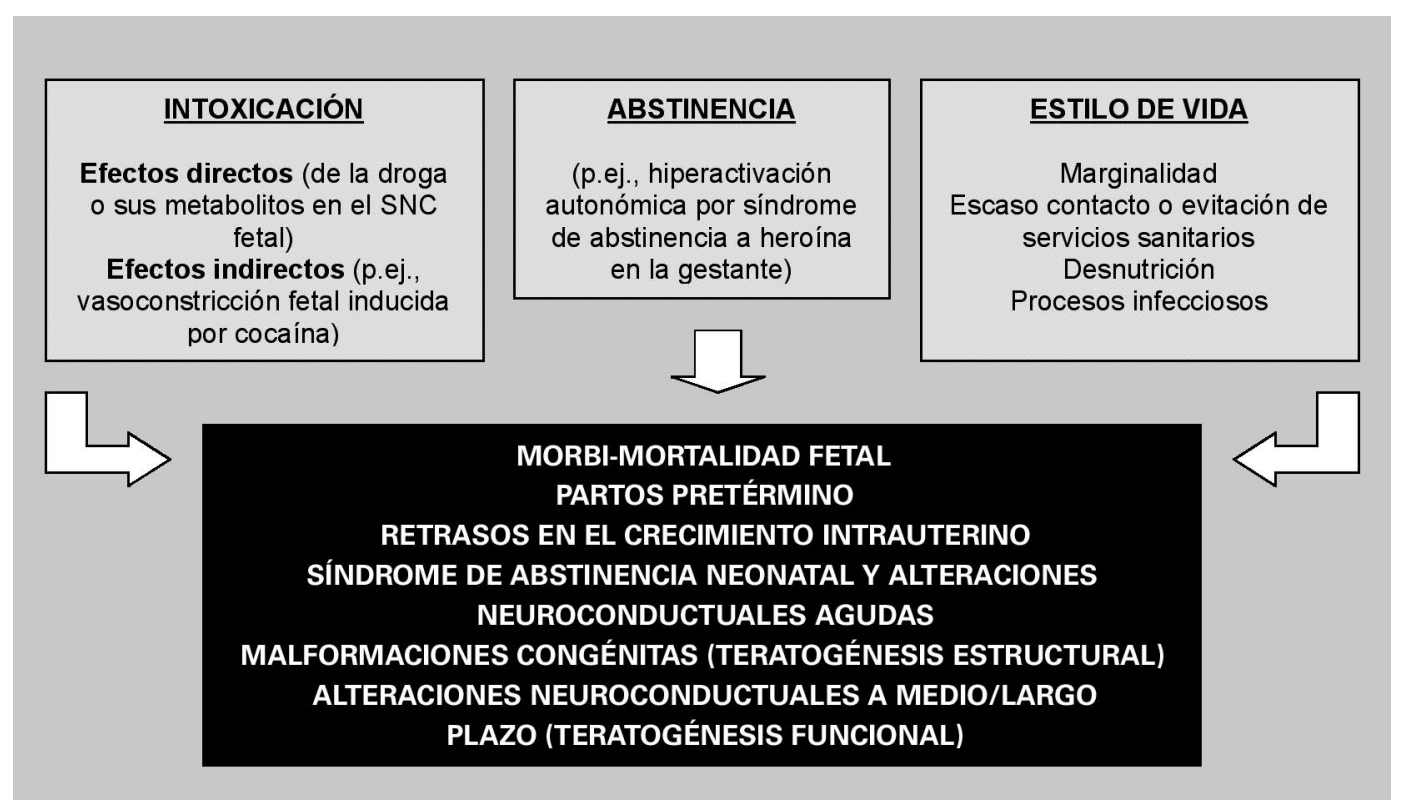

FIGURA 2: Efectos del consumo de drogas por la gestante 
tiva posterior cuando se emplea durante el primer año de vida ${ }^{26}$. En cuanto al momento en que se realiza la evaluación, una de las mayores críticas efectuadas en el ámbito de la exposición prenatal a sustancias radica precisamente en que la mayor parte de estudios se centran en las primeras semanas, meses o incluso años de vida ${ }^{5}$, lo que podría producir un solapamiento entre los efectos agudos y subagudos de la exposición, y alteraciones más permanentes ${ }^{21}$. Asimismo, para determinados déficits podría existir un periodo de silencio clínico en el que dichos déficits serán difícilmente evaluables o detectables ${ }^{4,27,28,}$, caso, por ejemplo, de las alteraciones en el funcionamiento ejecutivo. A ello se unen el hecho de que el modo de presentación de un determinado trastorno o efecto teratógeno puede variar a lo largo del desarrollo infantil ${ }^{28}$, y el importante papel que otro tipo de variables, como el entorno familiar y social, la calidad y estabilidad de lazos afectivos, estilos educativos inadecuados, experiencias de negligencia o maltrato, intervención de servicios sociales, etc, juegan sobre el desarrollo del niño y que serán comentados más adelante.

\section{EFECTOS DE LA EXPOSICIÓN PRENATAL A DROGAS}

\section{Alcohol}

El alcohol es una sustancia comprobadamente teratógena ${ }^{6}$, cuyo uso durante el embarazo puede dar lugar a un cuadro de embriofetopatía conocido como síndrome alcohólico fetal (fetal alcohol síndrome: FAS), caracterizado por ${ }^{8,29}$ :

- Presencia de retrasos en el crecimiento uterino y postnatal.

- Alteraciones del sistema nervioso central, entre las que pueden hallarse retraso mental (generalmente moderado), niveles bajos de atención, labilidad emocional y trastornos del comportamiento, irritabilidad, hiperactividad y alteraciones del desarrollo motor como hipotonía y déficits de la coordinación, etc.

- Alteraciones en la morfología craneofacial altamente distintivas, con presencia de microcefalia, anomalías palpebrales, labios delgados con hundimiento del labio superior, nariz chata y en "trompeta" con baja inserción del puente nasal, etc. En la práctica la presencia de estas anomalías, en especial cuando existe un consumo reconocido de alcohol durante el embarazo, son el indicio más claro de presencia de FAS. Con la edad la morfología facial tiende a mitigarse con persistencia de la microcefalia y de las diversas alteraciones neuroconductuales que concurren en el síndrome ${ }^{30}$.

Los estudios mediante técnicas de neuroimagen estructural encuentran que la microcefalia ligada al Síndrome Alcohólico Fetal no sólo corresponde a reducciones simétricas en el volumen cerebral sino que existen áreas anormalmente afectadas, tales como los ganglios basales, cerebelo, hipocampo y cuerpo calloso ${ }^{31}$. Del mismo modo, las técnicas de neuroimagen funcional revelan anomalías en la actividad eléctrica cerebral ${ }^{31}$

Sin embargo, actualmente se considera que el uso de alcohol repercute en el feto más allá de la manifestación clínica del síndrome alcohólico fetal. En 1996 el Instituto de Medicina Norteamericano propuso el uso de una nueva terminología para hacer referencia a aquellos cuadros que no cumplen criterios de FAS pero en los que existe una historia documentada de exposición prenatal al alcohol, bien mediante consumo regular, bien mediante episodios de ingesta masiva episódica, acompañados de algún tipo de manifestación clínica en el niño. Es por ello que durante los últimos años se hace referencia habitual en la literatura a los conceptos de defectos de nacimiento relacionados con el alcohol (alcohol-related birth defects: ARBD), y de desórdenes del neurodesarrollo relacionados con el alcohol (alcohol-related neurodevelopmental disorder: ARND) ${ }^{29}$. Actualmente no existe una dosis que pueda considerarse segura y dentro de cuyos márgenes no exista riesgo de afectación fetal.

Es interesante señalar en este punto que la existencia de un diagnóstico de síndrome alcohólico fetal no es una variable determinante para la presencia de diversas alteraciones funcionales y neuroconductuales, de modo que niños expuestos prenatalmente al alcohol, no diagnosticados de FAS, tienen ejecuciones similares en diversas pruebas y parámetros ${ }^{32-35}$. Entre las alteraciones más frecuentemente encontradas en ambos tipos de poblaciones se hallan las de tipo atencional ${ }^{35,36}$, lo que ha llevado a explorar la conexión entre exposición prenatal al alcohol y déficit de atención con hiperactividad, aunque la equivalencia de ambos cuadros sigue actualmente en entredicho ${ }^{36}$. Los problemas de conducta y la pobreza en las competencias psicosociales son también un hallazgo frecuente ${ }^{30,33,35}$. Las funciones ejecutivas suelen hallarse también afectadas, de manera que los niños prenatalmente expuestos a alcohol demuestran dificultades en habilidades de planificación y formación de conceptos y déficits en flexibilidad cognitiva ${ }^{30,32,34}$, así como déficits en la capacidad para modificar la conducta ante cambios en las condiciones de refuerzo y mayor variabilidad en la extinción estímulo-refuerzo, que dependen asimismo de las funciones ejecutivas y que en algunos casos correlacionan bien con la presencia de alteraciones conductuales ya mencionadas ${ }^{34}$. 
En referencia a los mecanismos para el desarrollo de daño neuronal inducido por alcohol en el cerebro en desarrollo, el consumo de alcohol por la gestante se ha relacionado con la inducción de apoptosis, situaciones de estrés oxidativo, alteración de los factores de crecimiento y migración neuronal, alteración de los sistemas fetales de neurotransmisión, alteraciones en el transporte de glucosa, y excitotoxicidad inducida por hiperactivación del receptor NMDA y entrada masiva de calcio intracelular en situaciones de abstinencia ${ }^{37}$.

Asimismo, existen diversos factores implicados en el desarrollo de afectación fetal y que se corresponden con los anteriormente mencionados para cualquier agente teratógeno, tales como la dosis y el patrón de consumo, el timing o periodo en que se produce la ingesta, diferencias metabólicas y diferencias en la susceptibilidad genética ${ }^{10}$. La dosis resulta un tema de especial relevancia ya que todavía no se ha encontrado la dosis "segura" por debajo de la cual no exista riesgo para el feto, por lo que la ingesta de bebidas alcohólicas está contraindicada en cualquier medida durante el embarazo. En referencia al momento en que se produce la ingesta, existe acuerdo en que el periodo crítico para las anomalías craneofaciales está en el primer trimestre ${ }^{1,8}$, mientras que el retraso en el crecimiento uterino cobra especial relevancia durante los dos segundos trimestres, especialmente durante el último dada la ganancia de peso que se produce en ese periodo. El grado de afectación neurológica también parece correlacionar con consumos más tardíos a lo largo del periodo de gestación, especialmente cuando se hace referencia a determinados parámetros neuroconductuales ${ }^{1}$, por lo que el cese de la ingesta alcohólica en cualquier punto a lo largo del embarazo, si bien no evita la aparición de daños producidos al inicio de la gestación (fundamentalmente malformaciones), sí posee valor en orden a la reducción de dicha afectación ${ }^{8}$.

Un aspecto especialmente interesante es el patrón de consumo de la madre y su relación con la afectación fetal ${ }^{10}$. Existen evidencias obtenidas a partir de la experimentación animal de que los episodios de ingesta abusiva en cortos periodos de tiempo son especialmente nocivos para el cerebro en desarrollo, y cuando dicha variable se ha explorado en humanos, los resultados también indican que el consumo en binges es un buen predictor de diversos parámetros de afectación neuroconductual a lo largo de la infancia ${ }^{10}$. La exposición fetal a episodios de importante ingesta alcohólica no sólo somete al feto a elevadas concentraciones de alcohol sino a sucesivos episodios de abstinencia que, como ya se ha comentado, pueden jugar un importante papel en el desarrollo de daño neuronal. Ello subraya la necesidad de contemplar en el rastreo del consumo de bebidas alcohólicas (y de otras sustancias), la especificación de cantidad de alcohol máxima consumida por ocasión. Este aspecto de la valoración es habitual en los servicios de atención en drogodependencias y busca evitar, precisamente, que un consumidor esporádico -en este caso consumidora-, sea erróneamente clasificado como de bajo riesgo atendiendo a la cantidad total de alcohol ingerida con respecto a un consumidor continuo ${ }^{10}$.

El consumo de alcohol por la gestante puede precipitar en el recién nacido un síndrome de abstinencia de corta latencia de aparición, relacionado con la ingesta de alcohol en momentos cercanos al parto ${ }^{8} \mathrm{y}$ caracterizado por irritabilidad, diaforesis, alteraciones en los patrones de sueño y alimentación y temblores $^{38}$. El tratamiento es sintomático.

\section{Psicoestimulantes}

La mayor parte de la investigación referida a los efectos de la exposición prenatal a estimulantes han utilizado la cocaína, fundamentalmente en forma de crack, siendo más infrecuente la atención prestada a otros psicoestimulantes. Los primeros estudios procedentes de Estados Unidos, debida -o indebidamente- amplificados por los medios, favorecieron la consideración de que la exposición prenatal a cocaína generaba daños graves e irreversibles en el neonato. Este fenómeno, conocido como el de los "crack kids" (niños del crack) llegó a ser una epidemia más mediática que real y actualmente numerosos autores siguen insistiendo en la necesidad de superar dicho mito en beneficio de una visión más científica y ajustada a los resultados de investigación ${ }^{5,16,39}$. En cualquier caso la mayor parte del volumen de investigación sigue realizándose en Norteamérica, siendo el crack la modalidad de consumo de cocaína mayormente estudiada y con una mayor representación en las muestras de mujeres afroamericanas, por lo que deben mantenerse las consiguientes cautelas a la hora de extrapolar datos tanto a otras poblaciones, como a diferentes modalidades de consumo ${ }^{23}$

La cocaína se asocia a un elevado número de complicaciones obstétricas, hecho que ha llevado a algunos autores a recomendar la exploración explícita del consumo de esta droga en gestantes heroinómanas ${ }^{40}$, dada además la elevada frecuencia del policonsumo de ambas sustancias. Entre estas complicaciones pueden encontrarse riesgo de aborto en los primeros meses, riesgo de muerte intraútero, desprendimiento de placenta y placenta previa y prematuridad ${ }^{9,14,24}$. Este tipo de complicaciones se relacionan con el incremento en la contractibilidad uterina debida al consumo de cocaína por la gestante y a los importantes efectos hipertensivos y vasoconstrictores de esta sustancia. La afectación del flujo uterino disminuye el transporte de oxígeno y nutrientes a través de la placenta ${ }^{41}$, lo que se relaciona con restricción en el crecimiento fetal ${ }^{39}$, a 
lo que se añade el efecto anorexígeno de este tipo de sustancias. Otro riesgo derivado de la vasoconstricción por cocaína son los fenómenos de hipoxia intermitente que pueden conducir a lesiones cerebrales isquémicas en el nonato. Esta patogenia vascular es la que, asimismo, se considera causa de las malformaciones ligadas a la exposición prenatal a la cocaína, fundamentalmente del tracto urinario y digestivo, y por las que la cocaína aparece clasificada como teratógena de baja frecuencia ${ }^{6}$. El consumo de anfetaminas por la gestante también se relaciona con este tipo de complicaciones $^{42}$ dado que, como psicoestimulante, comparte las propiedades farmacológicas de la cocaína y del mismo modo que ésta induce vasoconstricción.

La microcefalia es también un hallazgo frecuentemente informado ${ }^{9}$, de importancia dada su posible relación con la presencia de compromiso neurológico; sin embargo algunos estudios no encuentran asimetrías en la restricción del crecimiento uterino inducido por cocaína ${ }^{39}$. Resulta interesante señalar, una vez más, la dificultad de separar los efectos debidos a diferentes sustancias; aunque la hipertonía muscular es, del mismo modo que la microcefalia, un hallazgo frecuente en neonatos expuestos a cocaína, dicho podría deberse al uso concomitante de otras sustancias (en este caso, tabaco) a lo largo del embarazo ${ }^{43}$.

La sintomatología neurológica del recién nacido expuesto prenatalmente a cocaína ha generado también cierta controversia. Existe un gran número de niños prenatalmente expuestos que no muestran ningún tipo de alteración neuroconductual apreciable, y la adecuación o no de utilizar el término abstinencia para hacer referencia a la sintomatología presentada por hijos de mujeres drogodependientes a sustancias no depresoras es discutible ${ }^{38}$, de modo que para algunos autores, las alteraciones neurológicas en dichos casos no son efecto de la retirada de la sustancia psicoactiva (abstinencia) sino de su presencia en el Sistema Nervioso Central. Algunos autores han propuesto la existencia de tres patrones de afectación neurológica propia de la exposición prenatal a cocaína: un primer patrón definido como "excitable" (en el que tendrían cabida la existencia de irritabilidad, alteraciones en los patrones de sueño e hiperreactividad al medio) un segundo patrón definido como "deprimido", y un tercer patrón mixto caracterizado por una marcada inestabilidad para modular el arousal. Esta variabilidad en los patrones de respuesta se ha relacionado con los efectos directos (en el primer caso) e indirectos (en el segundo) de la cocaína sobre el feto ${ }^{5}$, o bien, según otros estudios, a la combinación con los efectos fetales de otras drogas, como marihuana y alcohol ${ }^{44}$. En particular el patrón excitable parece ser asimismo dosis dependiente ${ }^{5}$, aun cuando todavía no existe acuerdo en si esta sintomatología es característica específica de la exposición a cocaína u otro tipo de psicoestimulantes.
Con respecto al término de teratogenicidad conductual, anteriormente discutido, sí parece probable que la cocaína intraútero produzca alteraciones en la función y maduración cerebral, aunque sigue generando dudas si el efecto se debe a neurotoxicidad directa de la cocaína o a los fenómenos hipóxico-isquémicos anteriormente mencionados. La experimentación con animales demuestra que la cocaína es neurotóxica para el cerebro en desarrollo $2,45,46$, con especial susceptibilidad del área prefrontal, en especial el giro anterior cingulado ${ }^{46,47}$. A nivel humano, los hallazgos son menos concluyentes. Se han encontrado patrones anómalos de actividad cerebral con alteraciones en el EEG y otras medidas electrofisiológicas que podrían ser indicativas de menor conectividad interhemisférica, agregación neuronal y maduración cerebral 4,9,19. También se han hallado incrementos en los valores de creatinina frontal en neonatos expuestos a cocaína, posiblemente relacionados con alteraciones metabólicas secundarias a episodios múltiples de hipoxia uterina $^{48}$, en ausencia de cambios apreciables en marcadores de integridad neuronal. Ocasionalmente se ha informado de compromisos estructurales diversos relacionados en muchas ocasiones con infartaciones o hemorragias cerebrales ${ }^{4}$.

La relevancia clínica de este tipo de observaciones es aún hoy desconocida ${ }^{19}$. A ello se añade el hecho de que los efectos a medio y largo plazo asociados a la exposición prenatal a cocaína son diversos y los resultados en muchas ocasiones, poco concluyentes ${ }^{16}$. Se han sugerido asociaciones entre la exposición prenatal a cocaína con alteraciones o retrasos en el desarrollo del lenguaje ${ }^{18,49}$, el procesamiento de información visual y auditiva ${ }^{50,51}$, déficits en la regulación del arousal y los procesos atencionales ${ }^{52}$, alteraciones en la expresividad emocional ${ }^{16}$ y decrementos sutiles en el $\mathrm{Cl}^{9,53}$. En cierta medida, parte de estos déficits podrían estar relacionados entre sí. El papel que la cocaína, de manera directa o indirecta, juega sobre los sistemas monoaminérgicos y la relación que éstos tienen a su vez con mecanismos de regulación del arousal y la conducta continúa siendo un área de especial interés para los investigadores $5,52,54$. Si a ello se une la afectación de estructuras como el giro cingulado anterior, que juega un importante papel en los procesos atencionales, el vínculo es, una vez más, lógicamente plausible. No obstante, todavía es necesaria la realización de estudios metodológicamente rigurosos que permitan establecer con claridad las relaciones entre la exposición prenatal a cocaína y esta serie de déficits.

\section{Opiáceos}

Tras un periodo inicial situado en los años 70 y 80, la exposición prenatal a opiáceos ha generado un volumen relativamente menor de investigación con 
respecto a otras sustancias psicoactivas ${ }^{55}$. En nuestro contexto este tema es sin embargo del mayor interés, ya que, en tanto que en Estados Unidos el prototipo de embarazo de alto riesgo en adictas es, teóricamente, el de mujer drogodependiente al crack (con mayor presencia en las muestras de investigación de mujeres afroamericanas), en nuestro medio el prototipo es el de mujer con la heroína como droga de abuso principal, con presencia relativamente frecuente de politoxicomanía. Sin embargo tampoco es inusual encontrar en muestras americanas abuso conjunto de estas dos sustancias.

El consumo activo de heroína durante el embarazo se asocia a complicaciones obstétricas, tales como abortos, bajo peso para la edad gestacional con disminución de la talla y del perímetro cefálico, y partos patológicos ${ }^{14,55}$. Asimismo la gestante heroinómana puede verse afectada a lo largo del embarazo de sintomatología de abstinencia de mayor o menor intensidad, que dado el importante componente de hiperactivación autonómica, es susceptible de causar sufrimiento fetal intermitente. Tras el nacimiento aparece un síndrome de abstinencia característico (ver Figura 3), que es con frecuencia utilizado como referencia a la hora de evaluar la sintomatología de abstinencia en neonatos de madre dependientes a sustancias psicoactivas $^{38}$. Básicamente se compone de alteraciones a nivel de sistema nervioso central, con presencia de sintomatología indicativa de disrregulación autonómica y gastrointestinal, y del mismo modo que ocurre con adictos adultos, varía en aparición, intensidad y evolución en función del opiáceo consumido. El tratamiento es sintomático y requiere evaluar cada caso particular, optándose en primer tér- mino por un tratamiento de soporte no farmacológico para, sólo en casos en los que se considere pertinente, dar paso a terapia farmacológica ${ }^{14,38}$. La evaluación de la abstinencia neonatal a opiáceos cuenta con instrumentos específicos que son de especial utilidad a la hora de valorar la evolución del cuadro, tales como la escala de Lipsitz, la de Finnegan, y la de Ostrea ${ }^{14,38}$. La intensidad del síndrome de abstinencia no parece correlacionar con el desarrollo a largo plazo del niño ${ }^{38}$.

Existe un acuerdo general en que, dadas las complicaciones asociadas a la clínica de la mujer heroinómana, el mejor tratamiento para la misma es el ingreso en un programa de mantenimiento con metadona mientras transcurre el embarazo. Esta opción también generará en el niño un síndrome de abstinencia al nacer, pero aparecerá de forma más tardía (2472 horas tras el parto) y será más prolongado. A pesar de ello el mantenimiento con metadona a lo largo de la gestación presenta para las pacientes embarazadas ventajas importantes, tales como un control prenatal periódico, mejora de la retención en el tratamiento, del estado nutricional y de la calidad de vida, y en usuarias de droga por vía parenteral evitan la administración intravenosa, fuente de infecciones y problemas sanitarios que constituyen factores de riesgo adicionales para el feto. La reducción progresiva de la dosis a lo largo del embarazo es una posibilidad a lo largo del embarazo es una posibilidad entre las semanas 12 y 24 de gestación y realizándose preferentemente en el medio hospitalario ${ }^{40}$. Dosis iguales o inferiores a 20 grs de metadona diaria parecen minimizar el riesgo de abstinencia neonatal pero podrían incrementar el de abandono de tratamiento, teniendo además en cuenta que el embarazo produce alteraciones en los nive-

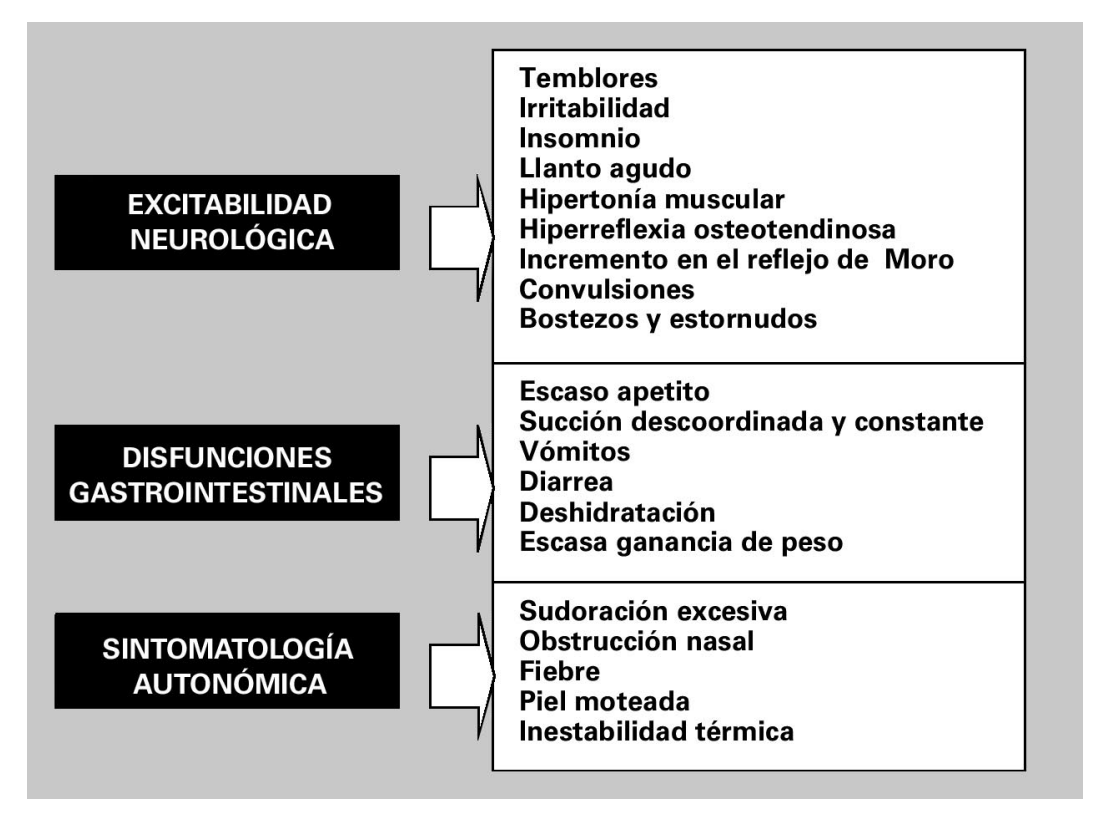

FIGURA 3: Síndrome de abstinencia neonatal 
les plasmáticos de opiáceos ${ }^{38}$. En los últimos años se está explorando el uso de otro tipo de agonistas opiáceos con especial atención a la buprenorfina, que parece producir buenos resultados ${ }^{56}$.

Los resultados sobre alteraciones a largo plazo derivadas de la exposición prenatal a opiáceos han sido contradictorias. Los modelos de laboratorio concluyen que la exposición prenatal a heroína altera los sistemas de neurotransmisión catecolaminérgicos y colinérgicos ${ }^{57}$, así como en los sistemas de respuesta al estrés ${ }^{58}$ mediados por los sistemas noradrenérgicos y opioide endógeno. La investigación con humanos, sin embargo, encuentra relativamente poca consistencia en la capacidad neuroteratógena de los opiáceos, evaluada a través de la existencia de déficits cognitivo-conductuales a medio y largo plazo. Parece existir cierto acuerdo en un retraso en el desarrollo psicomotor ${ }^{55,59}$ de significación clínica variable; el resto de problemáticas ligadas a los hijos de madre heroinómanas parece altamente dependiente del entorno ${ }^{55}$. Todo ello no desvaloriza la posible implicación de la exposición prenatal a opiáceos en la existencia de alteraciones neuroconductuales, pero sí refleja la posibilidad de un modelo de diatesis-estrés, que en mayor o menor media es aplicable a la exposición prenatal a las diversas sustancias de abuso aquí comentadas y en el que entran en juego variables adicionales a la exposición prenatal misma. Aunque estos aspectos serán comentados más adelante, se han encontrado datos indicativos de una mayor vulnerabilidad a entornos que podrían considerarse empobrecidos por parte de niños expuestos prenatalmente a opiáceos ${ }^{59}$.

\section{Tabaco}

El consumo de tabaco a lo largo del embarazo es relativamente frecuente en nuestra sociedad; asimismo, dicho consumo es menos susceptible, con respecto a otras sustancias de abuso, de verse reducido durante la gestación, y puede prolongarse más allá del parto facilitando la exposición pasiva del niño a esta sustancia ${ }^{60}$. Los hallazgos relativos a la exposición prenatal al tabaco son consistentes a la hora de encontrar una relación dosis-dependiente con el nacimiento de bebés con bajo peso ${ }^{60,61,62}$, posiblemente vinculado a los efectos anorexígenos e hipóxicos de la nicotina y a alteraciones en la función placentaria ${ }^{61}$. Alteraciones placentarias y partos pretérmino se han asociado también al consumo de tabaco por la gestante ${ }^{62}$. Durante los primeros meses de vida suele existir una recuperación del índice ponderal.

La exposición prenatal al tabaco también produce efectos neuroconductuales en el neonato, muchos de los cuales son asimismo dosis dependientes (hipertonía muscular, excitabilidad, alteraciones SNC y sin- tomatología gastrointestinal) ${ }^{63}$ y posiblemente reflejo de abstinencia nicotínica. La exposición durante el embarazo también es un conocido factor de riesgo de muerte súbita infantil y enfermedades respiratorias, del mismo modo que lo es la exposición postnatal al humo del tabaco 60,64 .

Del mismo modo que sucede con otras sustancias psicoactivas, la exposición prenatal a la nicotina genera alteraciones en algunos patrones de actividad cerebral como el EEG y los potenciales evocados al mes de vida que persiste hasta varios meses después ${ }^{19,65}$, y los estudios a medio plazo han asociado la exposición prenatal a tabaco con un amplio espectro de alteraciones con especial referencia a retrasos en el desarrollo del lenguaje, problemas de aprendizaje y memoria y déficits ejecutivos ${ }^{60,61,66}$, y problemas de conducta y negativismo infantil ${ }^{67,68}$. La exposición prenatal a la nicotina se ha llegado incluso, a relacionar con problemas de conducta, conducta antisocial, y delincuencia en la vida adulta ${ }^{60,61}$. Sin embargo, no todos estos estudios controlan la exposición prenatal a otras sustancias psicoactivas, lo que obviamente en este tipo de investigaciones resulta necesario, del mismo modo que resulta complejo valorar el impacto de variables ambientales. Con respecto a las alteraciones atencionales frecuentemente informadas en niños expuestos prenatalmente a sustancias psicoativas, es la exposición al tabaco durante la gestación la que se ha relacionado de manera más consistente con cuadros de déficits de atención con hiperactividad ${ }^{69}$.

Es interesante señalar aquí, asimismo, que la exposición pasiva al humo del tabaco por parte de la mujer embarazada produce también patrones de bajo peso y alteraciones en diversas funciones cognitivas, generalmente de menor intensidad que en el caso de fumadoras activas de manera que en este tipo de estudios, este factor debe ser controlado ${ }^{60}$.

La nicotina ha demostrado ser neuroteratógena a umbrales más bajos que los necesarios para producir alteraciones en el crecimiento fetal, produciendo efectos inhibitorios persistentes sobre la síntesis de ADN y pérdida neuronal irreversible ${ }^{2}$. Los estudios en animales de laboratorio señalan la presencia de alteraciones funcionales y estructurales en diversas zonas cerebrales $^{61}$ tales como déficits en la función dopaminérgica del córtex frontal y alteraciones estructurales del hipocampo (áreas CA3 y CA1)70,71. La relación entre exposición prenatal al tabaco, el desarrollo de anomalías estructurales o funcionales del SNC y la existencia de disfunciones cognitivas y conductuales de diverso tipo es biológicamente plausible ${ }^{66}$, en la misma medida en que lo es para otras sustancias de abuso y en especial dadas las extensas interacciones funcionales entre los sistemas adrenérgicos y catecolaminérgicos ${ }^{2}$. En este sentido se ha señalado que la peligrosidad de la exposición prenatal a nicotina ha 
sido sistemáticamente infravalorada con respecto a otro tipo de sustancias psicoactivas ${ }^{2}$.

\section{Cannabis}

El cannabis es posiblemente la droga ilegal más ampliamente consumida en numerosas poblaciones, frecuentemente asociada a policonsumos con alcohol $y$, sobre todo, tabaco. Dado que, como hemos señalado, estas dos sustancias tienen comprobados efectos negativos en el desarrollo fetal, los datos obtenidos en investigación humana deben ser interpretados con cautela.

Los estudios con modelos animales asocian la exposición prenatal a cannabinoides con retraso del crecimiento uterino ${ }^{11}$, déficitis de memoria, déficits en habituación a estímulos novedosos, especialmente en la categoría visual ${ }^{2,73}$ y alteraciones en el comportamiento social y sexual ${ }^{11,72}$, que es uno de los hallazgos informados de forma más consistente. Algunos de estos resultados son sexo-dependientes, hecho también observado en los resultados de investigación con exposición prenatal a otras sustancias ${ }^{61}$, y que, al menos en el caso del cannabis, se han relacionado con interacciones con los sistemas hormonales materno y fetal ${ }^{72}$. Se sabe asimismo que los patrones de localización y características del sistema cannabinoide endógeno son diferentes en el cerebro adulto con respecto al cerebro en desarrollo ${ }^{74,75}$, sugiriendo una especial vulnerabilidad de determinadas áreas cerebrales (hipocampo y amígdala) a la exposición prenatal al cannabis ${ }^{75}$. A ello se une que el sistema cannabinoide endógeno parece jugar un papel neurotrófico en el cerebro en desarrollo ${ }^{74}$, de modo similar al de otros sistemas de neurotransmisión.

En lo que se refiere al consumo de cannabis por parte de la mujer gestante, puede señalarse que existen resultados contradictorios al respecto ${ }^{76}$. Algunos autores asocian la exposición prenatal a cannabis con complicaciones obstétricas, inmadurez fetal y tendencia a la prematuridad, así como alteraciones neurológicas que se han interpretado como de abstinencia, tales como irritabilidad, inquietud y alteraciones del sueño y del apetito ${ }^{77}$. No obstante, dado que la misma existencia y/o relevancia de una abstinencia física al cannabis en consumidores adultos ha sido objeto de discusión durante años, una vez más la sintomatología podría ser indicativa de la acción de la sustancia sobre el SNC, o estar contaminada por la presencia de policonsumos en la mujer gestante, algo también aplicable a las complicaciones obstétricas observadas.

Resulta interesante señalar la relación entre exposición prenatal a cannabinoides con alteraciones en la habituación visual a edades tempranas y su asociación con un posible déficit en la maduración del sistema visual ${ }^{77}$. También se han encontrado menor rendimiento en pruebas de rendimiento verbal en determinadas edades, pero no en otras ${ }^{27}$, así como alteraciones persistentes en la función cerebral y los patrones de sueño65,78, y especialmente, déficits en tareas que implican a las funciones atencionales y ejecutivas y que implican tareas de atención sostenida y el manejo e integración de información visual ${ }^{79,80}$. Sin embargo, como ya se ha señalado, no todos los datos de investigación son consistentes ${ }^{27,76}$. Algunos autores señalan que la fuente estas divergencias en los resultados de diversos estudios, además del uso concomitante de otras drogas, podría ser la insensibilidad de los test para evaluar las disfunciones cognitivas sutiles ligadas a la exposición prenatal al cannabis o la dificultad de evaluar determinadas áreas de funcionamiento en edades tempranas ${ }^{27}$, caso del funcionamiento ejecutivo, al que dicha exposición parece hallarse ligado con cierta consistencia ${ }^{80}$.

\section{MDMA.}

En los últimos años se ha prestado una especial atención al uso de las llamadas drogas de síntesis y a su potencial neurotóxico sobre los terminales serotonérgicos. Entre ellas destaca la 3,4 metilendioximetanfetamina (MDMA o éxtasis). Los modelos animales encuentran diversas alteraciones bioquímicas y neuroconductuales en las crías expuestas prenatalmente a MDMA tales como déficits en el metabolismo monoaminérgico y dificultades de aprendizaje y memoria 81,82 ; sin embargo, no parece existir un patrón de neurotoxicidad equiparable al exhibido en cerebros desarrollados ${ }^{83}$. A ello debe unirse la especificidad de los patrones de consumo de este tipo de sustancias en humanos, caracterizadas por un uso generalmente episódico y autolimitado, y en muchas ocasiones combinado con el consumo o abuso de otras sustancias psicoactivas, algunas de ellas con marcados efectos sobre el feto como el alcohol y los psicoestimulantes ${ }^{84}$

\section{Benzodiacepinas.}

El uso no controlado de benzodiacepinas es frecuente en mujeres y su consumo es elevado tanto en la población general, como en la drogodependiente. Los primeros estudios sobre el potencial teratógeno de las benzodiazepinas, muchos de los cuales se centraban en el diazepam, señalaban la existencia de malformaciones diversas, tales como anomalías craneofaciales y hendiduras palatinas ${ }^{14,85-87}$ asociadas a su uso durante el primer trimestre de embarazo. Estudios más recientes señalan que dicha asociación, de existir, es de baja frecuencia ${ }^{14,85,87}$. Asimismo el uso de benzo- 
diazepinas durante el tercer trimestre de gestación, y especialmente en las semanas previas al parto, se asocia a sintomatología de abstinencia neonatal, similar a la producida por opiáceos y de intensidad variable ${ }^{14,38}$, así como a la posible presencia de un síndrome hipotónico denominado floppy infant o lactante laxo ${ }^{85,88}$. Éste último cuadro es informado con menor frecuencia, posiblemente por la falta de diagnóstico en casos más leves ${ }^{88}$. A medio plazo la exposición prenatal a benzodiacepinas incluso a dosis terapéuticas parece comprometer el desarrollo motor, especialmente fino, de los niños prenatalmente expuestos ${ }^{86}$, aunque los efectos en el desarrollo infantil a largo plazo son todavía desconocidos ${ }^{85}$, máxime considerando los factores que pueden presentarse de manera concomitante al abuso de benzodiazepinas (trastorno mental materno, abuso de otras drogas, etc).

\section{Cafeína.}

La cafeína está clasificada como teratógena en animales de laboratorio a dosis significativamente superiores a las consumidas habitualmente por humanos, por lo que en éstos, la ingesta de cafeína se realiza habitualmente dentro del rango no tóxico ${ }^{6}$. Cuando el consumo de cafeína por parte de la mujer gestante es extremadamente elevado se han encontrado tanto bajo peso al nacimiento ${ }^{89}$ como sintomatología consistente con la existencia de un síndrome de abstinencia neonatal ${ }^{38}$. A pesar de ello no existen indicios de que el consumo de cafeína en humanos produzca algún tipo de alteración estructural o funcional persistente.

\section{Inhalantes.}

El abuso de inhalantes es relativamente poco frecuente en nuestro medio. A pesar de que existe poca investigación en esta área, la exposición prenatal a inhalantes tales como gasolina o tolueno, parece relacionarse con retrasos en el crecimiento uterino, sintomatología de abstinencia, anomalías craneofaciales y esqueléticas, y retrasos en diversas áreas del desarrollo durante la primera infancia ${ }^{6,90}$.

\section{FACTORES PSICOSOCIALES.}

Como ya se ha señalado, el tipo y número de variables que pueden asociarse de un modo u otro con el resultado de la exposición prenatal a drogas es elevado 7,14,16,27,55. Estos extremos son especialmente relevantes cuando se abordan seguimientos a largo plazo, dado que es indudable que, a medida que el niño crece, aumenta el impacto que variables postnatales ajenas al estatus de exposición prenatal tienen sobre su desarrollo ${ }^{27}$. Este tipo de factores son en su mayor parte de carácter psicosocial.

El consumo de drogas tras el embarazo y durante la infancia del niño es una de las variables a considerar, ya que puede afectar a la capacidad de la madre de responder a las necesidades físicas y emocionales

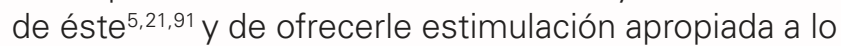
largo de su desarrollo ${ }^{25}$. En este sentido, un aspecto que se ha señalado como de gran importancia para el desarrollo psicoafectivo del niño a largo plazo es el establecimiento de un vínculo adecuado con el cuidador primario, generalmente, la madre. La calidad de este vínculo de apego, que se construye a través de una interacción de carácter diádico ${ }^{91}$, será buena en la medida en que ambos miembros de la díada emitan señales claras y consistentes, y sean capaces de interpretar las señales emitidas por el otro. Los efectos farmacológicos de las diversas sustancias y los ciclos de intoxicación-abstinencia pueden afectar a la interacción materno-infantil, al producir en la madre alteraciones perceptivas, afectivas y conductuales diversas, y pudiendo reducir tanto su sensibilidad para percibir e interpretar las claves emitidas por el niño, como su habilidad para emitir claves apropiadas. Si a ello unimos la posibilidad de que el niño sea "difícil" por hallarse bajo los efectos agudos o subagudos de la exposición prenatal a una droga, o por presentar alteraciones neuroconductuales de diversa índole, la calidad del vínculo puede verse comprometida por ambos miembros de la díada. Existen numerosos estudios que han abordado el tema del apego en hijos de mujeres drogodependientes ${ }^{25,92,93}$. No obstante, el desarrollo de apegos inseguros o desorganizados puede ser explicada no sólo en base a la influencia que los efectos farmacológicos de la droga tienen sobre la madre y el niño, sino al elevado grado de disfuncionalidad que caracteriza el entorno vital de la mujer drogodependiente, ya que entornos caóticos y desorganizados en ausencia de drogodependencia también comprometen la calidad del apego ${ }^{25}$.

En lo que se refiere a los estilos parentales, la drogodependencia materna se ha asociado con estilos educativos rígidos, inconsistentes y/o amenazantes $25,91,94$, que a medio o largo plazo pueden producir alteraciones conductuales o afectivas de diverso grado en el niño. No obstante, existe cierta variabilidad en las muestras que podrían estar mediada por, al menos, dos factores. Uno de ellos es la pertenencia a un nivel socioeconómico bajo, que se ha relacionado de forma independiente con estilos educativos más rígidos y menor grado de estimulación apropiada para el desarrollo infantil ${ }^{95}$. En la medida en que gran número de las muestras de mujeres estudiadas pertenecen a entornos socialmente conflictivos, el efecto concreto de la drogodependencia podría que- 
dar diluido, dado que la acumulación de problemáticas psicosociales en la mujer drogodependiente se relaciona con peores actitudes parentales ${ }^{96}$. A pesar de ello, existe un acuerdo común en que el estilo de vida del drogodependiente y los efectos de la intoxicación afectan negativamente a las habilidades educativas. La intoxicación por alcohol, por ejemplo, parece producir interacciones inconsistentes y desorganizadas con los menores, así como una disminución en la percepción parental de las conductas disruptivas del niño ${ }^{97}$.

El segundo aspecto que podría mediar la relación entre drogodependencia, apego, déficits en habilidades y estilos parentales y, a un nivel más amplio, entre drogodependencia y pronóstico en los hijos de drogodependientes, es la presencia de sintomatología psiquiátrica comórbida. En ausencia de trastorno adictivo, las madres afectadas de trastornos psicóticos o afectivos parecen poseer menor responsividad emocional hacia el niño y las madres depresivas parecen ser más hostiles e inconsistentes en su relación con éste ${ }^{98}$. Dada que la presencia de patología dual puede llegar a ser muy elevada y que las madres afectadas de trastornos mentales en ausencia de drogodependencia pueden considerarse por sí mismas un colectivo vulnerable $e^{98-100}$, se trataría en estos casos de un doble hándicap para la madre y para el niño. En efecto, la presencia de alteraciones diversas en los Ejes I (por ejemplo, trastornos de corte afectivo o psicótico) y II (trastorno antisocial o límite) según DSM parece relacionarse con peores habilidades parentales y/o peor pronóstico infantil ${ }^{95,101,102}$.

Quizá uno de los factores más frecuentemente señalados, y ya mencionados en estas líneas, como mediador de la relación entre drogodependencia familiar y evolución infantil es la pertenencia a un entorno desorganizado o socialmente conflictivo ${ }^{5,95}$. Este tipo de entornos puede aparecer asociado a la drogodependencia desde sus inicios, a modo de factor de riesgo, pero también ser consecuencia a largo plazo de la evolución del propio proceso adictivo. En la medida en que la adicción se convierte en el centro de la vida del adicto, sus recursos físicos y emocionales quedan hipotecados, lo que compromete progresivamente otras áreas vitales, entre ellas la vida familiar y el cuidado infantil. La relación entre drogodependencia parental y situaciones de abuso o negligencia infantil, sea de tipo físico o emocional, no es lineal, pero no es infrecuente que cuando existe intervención por parte de los servicios de protección de menores uno o ambos padres tengan problemas de drogas. La situación se complica cuando se trata de madres adolescentes, madres solteras con escasos apoyos familiares y sociales, o madres inmigrantes en situaciones de desprotección ${ }^{5,95}$, siendo asimismo muy frecuente en la mujer drogodependiente tener un historial de victimización previo y/o concu- rrente a su proceso adictivo ${ }^{103-105}$, hasta el punto de que la exploración específica del historial de abusos o maltrato debería, según algunos autores, ser rutinaria en los servicios de atención a drogodependiente, del mismo modo que la exploración del consumo o abuso de sustancias debería serlo en los recursos de atención a víctimas de maltrato ${ }^{105}$. Este tipo de vivencias, pasadas o presentes, pueden afectar tanto a la relación afectiva que la mujer establece con el niño, como a sus pautas educativas con éste, y al entorno que familiar en el que ambos viven. Las experiencias vicarias o directas de victimización, la existencia de un entorno familiar de violencia familiar unilateral o cruzada, y las situaciones recurrentes de estrés a lo largo de la primera infancia pueden ser importantes fuentes de compromiso para el adecuado desarrollo infantil $^{106}$.

Este tipo de variables deben ser considerados factores adicionales de riesgo, compartidos por cualquier niño con historial materno de drogodependencia más allá del estatus de exposición durante el embarazo. El historial paterno de drogodependencia también es susceptible de generar este tipo de problemáticas. Sin embargo, existen algunos autores que señalan que los niños expuestos durante el embarazo serían más vulnerables a entornos conflictivos o empobrecidos ${ }^{54,59}$, por lo que la exposición a otros factores de riesgo sería desencadenante de algún tipo de problemática con mayor facilidad. Los modelos animales sugieren una alteración de los sistemas de respuesta al estrés derivados de la exposición prenatal a sustancias tales como el alcohol, la cocaína y la heroína ${ }^{15,58,107}$, por lo que esta hipótesis no es desdeñable.

\section{INTERVENCIÓN EN LA MADRE DROGODEPEN- DIENTE.}

La experiencia de la gestación y la maternidad es un aspecto que suele asociarse con una mayor motivación para la búsqueda de tratamiento ${ }^{104,108-111}$ y con una toma de contacto con algún servicio sanitario que pueda aprovechar dicha motivación ${ }^{108,112}$. No obstante, la representación mental que la mujer tiene acerca de la maternidad, las expectativas que posee sobre sí misma en su rol de madre y las reacciones emocionales ante la perspectiva de la maternidad varían con cada mujer e, incluso, con cada embarazo para la misma mujer ${ }^{112}$. No es infrecuente encontrar sentimientos cruzados de apego, ansiedad y rechazo hacia el neonato, lo que unido al miedo a la estigmatización social, produzca un alejamiento de los servicios sanitarios y sociales más que un acercamiento a los mismos. Además, la gestación y la maternidad constituyen un importante factor de estrés y supone 
una serie de reajustes en la mujer que pueden agravarse en situaciones psicosociales conflictivas, como es la presencia de una adicción ${ }^{113}$. A pesar de ello, la preocupación por la salud del futuro hijo es una manifestación frecuente en la drogodependiente gestante $\mathrm{e}^{113,114}$ y posible motivo de diversas estrategias de control por parte de la misma ${ }^{113,115}$, entre las que se cuenta la toma de contacto con servicios prenatales. No obstante, los servicios de ginecología y obstetricia carecen a menudo de la formación necesaria para detectar problemas de drogas o de abordarlos de manera adecuada con la paciente ${ }^{108,109,116}$. A pesar de que las verbalizaciones abiertas de carácter negativo son poco frecuentes, la existencia de creencias disfuncionales en los profesionales sanitarios acerca del colectivo de drogodependientes y/o su posibilidad de recuperación ${ }^{108,109}$, añadidos a la mencionada falta de formación y al estilo defensivo y hostil con que este tipo de pacientes afrontan su implicación con los servicios sanitarios ${ }^{108,114}$, puede conducir a una interacción entre el profesional y la paciente que confirme las expectativas negativas de ambos y que se convierte en un círculo vicioso de difícil ruptura 113,115,117. En general existe acuerdo en que las aproximaciones a la cuestión de tipo confrontativo, rígido y en algunos países, claramente persecutorias, no son constructivas para la madre ni para el niño ${ }^{118}$. El enfoque más apropiado suele considerarse en el ámbito de las aproximaciones de corte motivacional, no confrontativas y en contactos sucesivos ${ }^{20}$, en la medida en que lo permita la situación sociosanitaria de la mujer y del feto. Debe considerarse en cualquier caso, que la mujer drogodependiente puede optar por estrategias alternativas al tratamiento de la adicción y que caerían dentro de un enfoque de "reducción del daño"115 tales como la disminución en el consumo de una sustancia, su sustitución por otra teóricamente "menos peligrosa", o la introducción de cambios en el estilo de vida. El beneficio de estos enfoques cuando se trasladan a un contexto profesional debe ser tenido en cuenta, en la medida en que la insistencia en la abstinencia completa puede ser ineficaz e incluso contraproducente $^{115}$, y dado que es preferible retener a la mujer en el marco de un contacto sociosanitario continuado que garantice el cuidado prenatal.

Por otro lado, las responsabilidades derivadas de la maternidad constituyen a menudo un arma de doble filo, habida cuenta que, aunque motivan para la rehabilitación, compiten por los recursos de la mujer, en ocasiones en momentos especialmente críticos del proceso terapéutico ${ }^{119}$. En este sentido se produce un paralelismo con lo que se observa en el tratamiento de enfermos mentales que son, a su vez, padres: los profesionales tienen a tratar al paciente como unidad olvidando la diversidad de roles que éste desempeña y entre los cuales la maternidad (o paternidad) juega un importante papel ${ }^{110}$. En el caso de la mujer, la pre- sión social orientada al desempeño "adecuado" del rol de madre, el sentimiento de fracaso como tal, o la incapacidad de mantener un contacto apropiado con los hijos cuando se le ha retirado la tutela, constituye una importante fuente de estrés y puede precipitar eventualmente una recaída91,120, o producir estancamientos en el proceso de recuperación de aquellas mujeres inmersas en tratamientos no siempre sensibles a estos aspectos.

A este respecto es especialmente interesante subrayar que la evaluación que la mujer hace de sus habilidades y capacidades como madre están mediadas, no sólo por la interacción materno-filial, sino por el momento que atraviesa en su proceso adictivo y los mensajes que recibe de los profesionales sanitarios, la sociedad y la red social de apoyo ${ }^{21}$. Estableciendo una vez más un paralelismo con aquellas mujeres afectadas de otros trastornos mentales la presencia de un compañero emocionalmente estable y un entorno familiar y social de soporte resulta un importante factor de protección. Sin embargo, en el ámbito de las adicciones, la mujer suele presentar un menor apoyo que el hombre a la hora de acudir a tratamiento ${ }^{121,122 ;}$ en muchas ocasiones el papel del varón resulta importante e incluso determinante en el inicio y evolución del proceso adictivo de la mujer ${ }^{104,111,122}$, por lo que no es raro que ambos progenitores se hallen inmersos en problemas con las drogas, y las relaciones con la familia de uno u otro pueden sufrir importantes deterioros que mengüen la capacidad de prestar apoyo constructivo en el ejercicio de roles parentales. Estos aspectos son especialmente críticos en la medida en que las responsabilidades y cargas familiares siguen siendo actualmente más "propias" de la mujer ${ }^{122}$, y dado que para muchos autores, el proceso de socialización de la mujer y la construcción de la identidad femenina se produce en parte, a partir de su capacidad para crear y mantener lazos de afiliación con el otro ${ }^{122,123}$. Existe a menudo un complejo solapamiento entre las redes sociales de apoyo y de consumo en la mujer drogodependiente ${ }^{124}$, en la medida en que el uso compartido de drogas se usa para mantener dichos lazos ${ }^{122} \mathrm{O}$ que las figuras de apoyo facilitan de manera directa o inadvertida la continuidad del proceso adictivo ${ }^{124}$. Se hace necesario, por tanto, una cuidada exploración de estos aspectos, la intervención sobre dichas figuras, cuando fuera posible, o el abordaje con la paciente de cómo vive estas dinámicas relacionales, en especial las de corte afectivo-sexual, dado que juegan un importante papel en su rehabilitación y la mujer tiende a repetirlas a lo largo de su trayectoria vital ${ }^{123}$.

Un ejemplo especialmente relevante en este ámbito es la implicación de la familia extensa, generalmente abuelos, en el cuidado de los hijos durante las fases activas del proceso adictivo ${ }^{114,117,120,125}$. Gran parte de estos acogimientos son "de hecho" o no formalizados ${ }^{125}$ y en no pocas ocasiones pueden suponer 
dificultades adicionales a los acogimientos en familia educadora, al generar esta situación problemas de identificación de roles -especialmente cuando la madre sigue conviviendo en el domicilio-114,120 y verse contaminada por conflictos emocionales no resueltos derivados del desarrollo progresivo de la adicción en la hija. Si a ello se añade las experiencias disfuncionales que pudo haber vivido la mujer en el núcleo familiar a lo largo de la propia infancia ${ }^{91,114,119}$, las dificultades se multiplican. Todo ello lleva a la necesidad de incluir a la familia en el proceso de rehabilitación de la paciente y a examinar los reajustes que dicho proceso provoca en todos ellos ${ }^{119,120}$.

Es aquí necesario señalar que, a menudo, la rehabilitación de una drogodependencia es larga y costosa y los contactos con los hijos, conflictivos, poco frecuentes o directamente inexistentes. Puede darse el caso de que a lo largo de los años, la relación materno-filial se halla visto dañada y el niño haya establecido lazos afectivos sólidos con otras figuras de apego para garantizar su propia estabilidad y supervivencia. En este contexto el niño puede vivir como una intrusión o amenaza el retorno de una madre que nunca o rara vez estuvo presente en su vida ${ }^{120}$, dando lugar a situaciones altamente conflictivas. La intervención en estos casos debe incluir el manejo por parte de la mujer del resentimiento o alejamiento emocional del menor, así como la comprensión de éste, desde una perspectiva apropiada para su nivel evolutivo, de la problemática presentada por su madre. En casos extremos sería necesario para la mujer elaborar la comprensión y el duelo de la pérdida del rol de madre.

Por otro lado, la consideración de la existencia de una adicción como parámetro único de evaluación de las capacidades parentales de la mujer es inadecuado ${ }^{117}$, dado que la afectación de la competencia parental y la calidad en la relación materno-filial puede variar en grados y depender de otros factores. De modo similar, la rehabilitación de la drogodependencia no conlleva necesariamente una automática mejora de las habilidades parentales ${ }^{117}$. Se ha dado la curiosa paradoja de que la mejora de las habilidades educativas, incluso en ausencia de tratamiento específico de la drogodependencia, repercute positivamente en los problemas de los hijos de drogodependientes lo que, a su vez, influye positivamente en el estado afectivo y el consumo de drogas de la madre ${ }^{110}$. Por tanto, y en la línea de la "reducción de daños" anteriormente mencionada, no debe obviarse la importancia de cualquier tipo de intervención de carácter social, sanitario o educativo, que mejore a diversos niveles las condiciones de vida de la mujer y del niño. Por último, debe subrayarse una vez más que la necesidad de un enfoque multidisciplinar y de la coordinación entre recursos se hace especialmente evidente en situaciones como las aquí reflejadas ${ }^{116,117,121}$. Sucede de manera relativamente frecuente que dicha coordinación sea deficiente, bien por falta de tiempo y de medios, bien porque el abordaje de la problemática parte de diferentes filosofías o tiene diferentes objetivos ${ }^{116}$. En la medida en que diferentes recursos y profesionales transmiten información contradictoria o persiguen metas que pueden ser -o parecer- excluyentes, la situación de la madre drogodependiente y su hijo se ve negativamente afectada.

\section{CONCLUSIONES}

1. La mayor parte de drogas de abuso generan complicaciones obstétricas, derivadas tanto de los efectos de la droga como de las condiciones de vida de la gestante, y son susceptibles de producir sintomatología de disrregulación neurológica postparto. Muchos neonatos nacidos de madre drogodependiente con consumo activo durante el embarazo pueden, no obstante, no presentar sintomatología perceptible.

2. El consumo de diversos tipos de drogas por parte de la mujer gestante se ha asociado en la literatura científica de los últimos años, tanto a teratogénesis estructural (presencia de malformaciones) como funcional (alteraciones del SNC en ausencia de malformaciones evidentes y manifestadas con alteraciones neuroconductuales a lo largo del desarrollo infantil). Los resultados más sólidos de investigación hacen referencia a la exposición al alcohol, con atención al síndrome alcohólico fetal, los defectos de nacimiento relacionados con el alcohol, y los desórdenes del neurodesarrollo relacionados con el alcohol. Para otras drogas, los resultados han resultado a menudo contradictorios, pero parecen apuntar, en el campo de la teratogénesis funcional, a los déficits atencionales como los más frecuentemente asociados a la exposición prenatal a cocaína y nicotina, y los déficits en el desarrollo motor para opiáceos y benzodiacepinas. En la mayor parte de casos, por tanto, se trataría de alteraciones específicas de determinados dominios cognitivos y no tanto de alteraciones globales de funcionamiento.

3. Un aspecto que no ha sido abordado en estas líneas y que está siendo asimismo objeto de investigación es la capacidad potencial de la exposición prenatal a una droga para modificar la respuesta a ésta u otras drogas en la vida adulta. Existen datos derivados de modelos animales en los que la exposición preparto induce alteraciones en la conducta posterior de autoadministración de droga. 
4. La relación entre la droga y los déficits o problemáticas presentadas está en función de gran número de factores pre y postparto, entre los que se incluyen el tipo de droga, la dosis, timing, policonsumos, prematuridad, multiparidad, pobreza, disfuncionalidad familiar, abuso, negligencia, contacto con recursos sociales, etc. Con independencia del carácter teratógeno de la droga consumida, el embarazo de la mujer drogodependiente puede ser considerado de riesgo, lo que compromete el pronóstico del feto y del niño. Del mismo modo, la existencia de una drogodependencia instaurada por parte de los cuidadores primarios de un menor puede conducir a situaciones altamente desestructuradas y conflictivas en el seno de la familia (nuclear y/o extensa) durante la infancia de éste, lo que puede influir en la aparición de déficits de aprendizaje, problemas de conducta y emocionales, etc. En el caso de que haya existido exposición prenatal a algún tipo de droga, y de que de ésta se haya derivado algún tipo de déficit neuroconductual, la vulnerabilidad para el menor podría incrementarse. Ello dificulta enormemente la investigación, en la medida en que no todos los estudios comparten los mismos criterios metodológicos ni controlan o interpretan del mismo modo este tipo de variables. Durante los próximos años debería realizarse un esfuerzo en este sentido.

5. A la vista de los datos actuales, puede considerarse que el impacto de la exposición prenatal a una sustancia psicoactiva responde a un modelo complejo en el que todos estos factores se suman y/o interactúan para potenciar o mitigar las consecuencias a medio y largo plazo de dicha exposición. A pesar de que existe consenso en cuanto a este aspecto, el peso relativo de dichas variables y la naturaleza exacta de su relación está aún por dilucidar, lo que constituye una laguna importante que, de cubrirse, sería de gran relevancia de cara al establecimiento los programas de intervención más apropiados.

6. En lo que se refiere a dichas intervenciones, actualmente puede considerarse que la detección precoz, el establecimiento de canales fluidos de comunicación entre recursos, la formación de los diversos tipos de profesionales que entran en contacto con estos colectivos, y la investigación sobre aquellos factores que faciliten el contacto y retención de la mujer y madre drogodependiente en los recursos específicos de tratamiento deben ser consideradas objetivos prioritarios, acompañado de otro tipo de actuaciones de carácter social o educativo, que pueden resultar no sólo valiosas, sino necesarias, a la hora de garantizar que la intervención obtenga resultados positivos. La intervención sobre otros miembros de la familia, en especial el padre y la familia extensa, debería ser también prioritaria.

7. En especial en el momento del embarazo, se recomienda evitar enfoques condenatorios sobre la futura madre y realizar intervenciones de corte motivacional. Cuando se trata de intervenciones sobre familias ya formadas, debe recordarse el principio del "interés superior del niño", si que ello suponga, nuevamente, la condena o reproche automático sobre la familia. Aun cuando parezca reiterativo dado que se trata de una indicación clásica en el ejercicio de la práctica profesional, es obligatoria una cuidada evaluación de cada caso particular, en la que se exploren los aspectos concretos de la drogodependencia materna, el vínculo afectivo materno-filial, las pautas educativas, los apoyos sociales, la problemática presentada por el menor, etc. Todo ello considerando el aprovechamiento y desarrollo de las potencialidades de los individuos objeto de intervención.

\section{REFERENCIAS}

1. Coles CD. Critical periods for prenatal alcohol exposure: evidence from animal and human studies. Alcohol Health and Research World 1994; 18: 22-9.

2. Slotkin T. Fetal nicotine or cocaine exposure: which one is worse? J Pharmacol Exp Ther 1998; 285: 93145.

3. Pérez Landeiro A, Allende-Bandrés MA, Agustín Fernández MJ, Palomo Palomo P. Teratogénesis: clasificaciones. Farm Hosp 2002; 26: 171-77.

4. Kosofsky BE. The effect of cocaine on developing human brain. En: Kilbey MM, Asghar K, editors. Methodological issues in controlled studies on effects of prenatal exposure to drug abuse. NIDA Research Monograph $N^{\circ}$ 114. Rockville, MD: National Institute of Drug Abuse, 1991. p 128-43.

5. Lester BM, LaGasse L, Freier K, Brunner S. Studies of cocaine-exposed human infants. In: Wetherington $C L$, Smeriglio VL, Finnegan LP, eds. Behavioral studies of drug-exposed offspring: methodological issues in human and animal research. NIDA Research Monograph $N^{\circ}$ 164. Rockville, MD: National Institute on Drug Abuse, 1996, p 175-210.

6. Brent RL. The application of the principles of toxicology and teratology in evaluating the risks of new drugs for treatment of drug addiction in women of reproductive age. In: Chiang CN, Finnegan LP, eds. Medications development for the treatment of pregnant addicts and their infants. NIDA Research Monograph $N^{\circ} 149$. Rockville, MD: National Institute on Drug Abuse, 1995. p 130-84. 
7. Sokol RJ, Ager JW, Martier SS. Methodological issues in obtaining and managing substance abuse information from prenatal patients. In: Kilbey MM, Asghar K, eds. Methodological issues in epidemiological, prevention, and treatment research on drug-exposed women and their children. NIDA Research Monograph $\mathrm{N}^{\circ}$ 117. Rockville, MD: National Institute on Drug Abuse, 1992. p 80-97

8. Romera Modamio G, Fernández López A, Jordán García Y, Pastor Gómez A, Rodríguez Miguélez JM, Botet Mussons F, et al. Embriofetopatía alcohólica. Casuística neonatal propia en los últimos doce años. Anales Españoles de Pediatría 1997; 47:405-9.

9. Dow-Edwards D. Comparability of human and animal studies of developmental cocaine exposure. In: Wetherington CL, Smeriglio VL, Finnegan LP, eds. Behavioral studies of drug-exposed offspring: methodological issues in human and animal research. NIDA Research Monograph No 164. Rockville, MD; National Institute on Drug Abuse, 1996. p 146-74.

10. Maier SE, West JR. Drinking patterns and alcoholrelated birth-defects. Alcohol Res Health 2001; 25: 168-74.

11. Abel EL. Effects of prenatal exposure to cannabinoids. In: Pinkert T, ed. Current research on the consequences of maternal drug abuse. NIDA Research Monograph $N^{\circ}$ 59. Rockville, MD: National Institute on Drug Abuse, 1985. p 20-35.

12. Poch Olivé ML. Neurobiología del desarrollo temprano. Contextos Educativos 2001; 4: 79-94.

13. Herlenius E, Lagercrantz $H$. Neurotransmitters and neuromodulators during early human development. Early Hum Dev 2001; 65: 21-37.

14. Solís Sánchez G, Solís Sánchez JL, Díaz González T. Exposición prenatal a drogas y efectos en el neonato. Trastornos Adictivos 2001; 3: 256-62.

15. Spear LP. Assessment of the effects of developmental toxicants: pharmacological and stress vulnerability in offspring. In: Wetherington CL, Smeriglio VL, Finnegan $L P$, eds. Behavioral studies of drug-exposed offspring: methodological issues in human and animal research. NIDA Research Monograph No 164. Rockville, MD: National Institute on Drug Abuse, 1996.p 125-45.

16. Frank DA, Augustyn M, Knight WG, Pell T, Zuckermann B. Growth, development and behaviour in early childhood following prenatal cocaine exposure. A systematic review. JAMA 2001; 285: 1613-25.

17. Kwong TC, Ryan RM. Detection of intrauterine illicit drug exposure by newborn drug testing. Clin Chem 1997; 43: 235-42.

18. Singer LT, Arendt R, Minnes $S$, Salvator A, Siegel AC, Lewis BA. Developing language skills of cocaineexposed infants. Pediatrics 2001; 107: 1057-64.

19. Scher MS, Richardson GA, Day NL. Effects of prenatal cocaine/crack and other drugs exposure on electroencephalographic sleep studies at birth and one-year. Pediatrics 2000; 105: 39-48.

20. Handmaker NS, Wilbourne P. Motivational interventions in prenatal clinics. Alcohol Res Health 2001; 25: 21929.

21. Coles CD. Discussion: measurement issues in the study of effects of substance abuse in pregnancy. In: Kilbey MM, Asghar K, eds. Methodological issues in epidemiological, prevention, and treatment research on drug-exposed women and their children. NIDA Research Monograph N ${ }^{\circ}$ 117. Rockville, MD: National Institute on Drug Abuse, 1992. p 248-58.

22. Martín Mandomingo MA, Solís Sánchez G, Málaga Guerrero S, Cuadrillero Quesada C, Pérez Méndez C, Matesanz Pérez JL. Consumo de drogas durante el embarazo y morbilidad neonatal: cambios epidemiológicos en los últimos 10 años. An Pediatr 2003; 58: 574-9.

23. Chassnoff IJ. Methodological issues in studying cocaine use in pregnancy: a problem of definitions. In: Kilbey MM, Asghar K, eds. Methodological issues in controlled studies on effects of prenatal exposure to drug abuse NIDA Research Monograph No 114. Rockville, MD: National Institute on Drug Abuse, 1991. p 55-65.

24. Bandstra ES. Assessing acute and long-term physical effects of in utero drug exposure on the perinate, infant, and child. In: Kilbey MM, Asghar K, eds. Methodological issues in epidemiological, prevention, and treatment research on drug-exposed women and their children. NIDA Research Monograph $\mathrm{N}^{\circ}$ 117. Rockville, MD: National Institute on Drug Abuse, 1992. p 212-27.

25. Mayes LC. Exposure to cocaine: behavioural outcomes in preschool and school-age children. In: Wetherington CL, Smeriglio VL, Finnegan LP, eds. Behavioral studies of drug-exposed offspring: methodological issues in human and animal research. NIDA Research Monograph No 164. Rockville, MD: National Institute on Drug Abuse, 1996. p 211-29.

26. Jacobson JL, Jacobson SW. Assessment of teratogenic effects on cognitive and behavioural development in infancy and childhood. In: Kilbey MM, Asghar K, eds. Methodological issues in controlled studies on effects of prenatal exposure to drug abuse. NIDA Research Monograph No 114. Rockville, MD: National Institute on Drug Abuse, 1991. p 248-61.

27. Fried PA. Behavioral outcomes in preschool and school-age children exposed prenatally to marijuana: a review and speculative interpretation. In: Wetherington CL, Smeriglio VL, Finnegan LP, eds. Behavioral studies of drug-exposed offspring: methodological issues in human and animal research. NIDA Research Monograph N 164. Rockville, MD: National Institute on Drug Abuse, 1996. p 242-60. 
28. Hans SL. Prenatal drug exposure: behavioural functioning in late childhood and adolescence. In: Wetherington CL, Smeriglio VL, Finnegan LP, eds. Behavioral studies of drug-exposed offspring: methodological issues in human and animal research. NIDA Research Monograph No 164. Rockville, MD: National Institute on Drug Abuse, 1996. p 262-76.

29. Warren KR, Foudin LL. Alcohol related birth defects: the past, present and future. Alcohol Res Health 2001; 25: 153-8.

30. Steinhausen $H$, Spohr HL. Long-term outcome of children with Fetal Alcohol Syndrome: psychopathology, behavior, and intelligence. Alcohol Clin Exp Res 1998; 22: 334-8.

31. Mattson S, Schoenfeld AM, Riley EP. Teratogenic effects of alcohol on brain and behavior. Alcohol Res Health, 2001; 25: 185-91.

32. Mattson SN, Goodman AN, Caine C, Delis DC, Riley EP. Executive functioning in children with heavy prenatal alcohol exposure. Alcohol Clin Exp Res 1999; 23: 1808-15.

33. Roebuck TM, Mattson SN, Riley EP. Behavioral and psychosocial profiles of alcohol-exposed children. Alcohol Clin Exp Res 1999; 23: 1070-6.

34. Kodituwakku PY, Kalberg W, May PA. The effects of prenatal alcohol exposure on executive functioning. Alcohol Res Health 2001; 25: 192-8.

35. Jacobson JL, Jacobson SW. Drinking moderately and pregnancy. Effects on child development. Alcohol Res Health 1999; 23: 25-30.

36. Coles CD, Platzman KA, Raskin-Hood CL, Brown RT, Falek A, Smith IE. A comparison of children affected by prenatal alcohol exposure and attention deficit, hyperactivity disorder. Alcohol Clin Exp Res 1997; 21 : 150-61.

37. Goodlett CR, Horn KH. Mechanisms of alcohol-induced damage to the developing nervous system. Alcohol Res Health 2001; 25: 175-84.

38. Committee on Drugs. Neonatal Drug Withdrawal. Pediatrics 1998; 101: 1079-88.

39. Bandstra ES, Morrow CE, Anthony JC, Churchill SS, Chitwood DC, Steele BW, et al. Intrauterine growth of full-term infants: impact of prenatal cocaine exposure. Pediatrics 200; 108: 1309-19.

40. Leza Cerro JC. Opiáceos (III) Tratamiento de la dependencia con agonistas opiáceos. En Lorenzo P, Ladero JM, Leza JC, Lizasoain L, eds. Drogodependencias. Farmacología, patología, psicología y legislación. Madrid: Editorial Médica Panamericana; 1999. p 77-86

41. Pastrakuljic A, Derewlany LO, Knie B, Koren G. The effects of cocaine and nicotine on amino acid transport across the human placental cotyledon perfused in vitro. J Pharmacol Exp Ther 2000; 294: 141 - 6.
42. Plessinger MA. Prenatal exposure to amphetamines: risks and adverse outcomes in pregnancy. Obst Gynecol Clin North Am 1998; 25: 119-38.

43. Dempsey DA, Hajnal BL, Partridge JC, Jacobson SN, Good W, Jones RT, et al. Tone abnormalities are associated with maternal cigarette smoking during pregnancy in in utero cocaine-exposed infants. Pediatrics 2000; 106: 79-85.

44. Napiorkowski B, Lester BM, Freier MC, Brunner S, Dietz L, Nadra A, et al. Effects of in utero substance exposure on infant neurobehavior. Pediatrics 1996; 98: 71-5.

45. Lidow M. Nonhuman primate model of the effect of prenatal cocaine exposure on cerebral cortical development. Ann N Y Acad Sci 1998; 846: 182-93.

46. Levitt P, Harvey JA, Friedman E, Simansky K, Murphy $\mathrm{EH}$. New evidences for neurotransmitter influences on brain development. Trends Neurosci 1997; 20: 26974.

47. Morrow BA, Elsworth JD, Roth RH. Axo-axonic structures in the medial prefrontal cortex of the rat: reduction by prenatal exposure to cocaine. J Neurosci 2003; 23: 5227-34.

48. Smith LM, Chang L, Yonekura ML, Gilbride K, Kuo J, Poland RE, et al. Brain proton magnetic resonance spectroscopy and imaging in children exposed to cocaine in utero. Pediatrics 2001; 107: 227-31.

49. Nulman I, Rovet J, Greenbaum R, Loebstein M, Wolpin J, Pace-Asciak P, et al. Neurodevelopment of adopted children exposed in utero to cocaine: the Toronto Adoption Study. Clin Invest Med 2001; 24: 129-37.

50. Potter SM, Zelazo PR, Stack DM, Papageorgiou A. Adverse effects of fetal cocaine exposure on neonatal auditory information processing. Pediatrics 2000; 105: e40. Disponible en: http://pediatrics.aappublications. org/cgi/reprint/105/3/e40 [consultado el 11/07/04].

51. Heffelfinger AK, Craft S, White DA, Shyken J. Visual attention in preschool children prenatally exposed to cocaine: implications for behavioral regulation. J Int Neuropsychol Soc 2002; 8:12-21.

52. Mayes LC, Grillon C, Granger R, Schottenfeld R. Regulation of arousal and attention in preschool children exposed to cocaine prenatally. Ann N Y Acad Sci. 1998; 846: 126-43

53. Lester BM, Lagasse LL, Seifer R. Cocaine exposure and children: the meaning of subtle effects. Science 1998; 282: 633-4.

54. Mayes LC. A behavioral teratogenic model of the impact of prenatal cocaine exposure on arousal regulatory systems. Neurotoxicol Teratol 2002; 24: 385-95.

55. Kaltenbach KA. Exposure to opiates: behavioural outcomes in preschool and school-age children. In: Wetherington CL, Smeriglio VL, Finnegan LP, eds. Behavioral studies of drug-exposed offspring: 
methodological issues in human and animal research. NIDA Research Monograph No 164. Rockville, MD: National Institute on Drug Abuse, 1996. p 230-41.

56. Schindler SD, Eder $H$, Ortner R, Rohrmeister $K$, Langer M, Fischer G. Neonatal outcome following buprenorphine maintenance during conception and throughout pregnancy. Addiction 2003; 98: 103-10.

57. Slotkin TA, Seidler FJ, Yanai J. Heroin neuroteratogenicity: delayed-onset deficits in catecholaminergic synaptic activity. Brain Res 2003; 984 : 189-97.

58. Vathy I. Prenatal opiate exposure: long-term CNS consequences in the stress system of the offspring. Psychoneuroendocrinology 2002; 27: 273-83.

59. Hans SL. Developmental consequences of prenatal exposure to methadone. Ann N Y Acad Sci 1989; 562: 195-207.

60. Cornelius MD, Day NL. The effects of tobacco use during and after pregnancy on exposed children. Relevance of findings for alcohol research. Alcohol Res Health 2000; 24:242-49.

61. Ernst M, Moolchan ET, Robinson ML. Behavioral and neural consequences of prenatal exposure to nicotine. J Am Acad Child Adoles Psychiatry. 2001; 40: 63041.

62. Werler MM. Teratogen update: smoking and reproductive outcomes. Teratology 1997; 55: 382-8.

63. Law KL, Stroud LR, LaGasse LL, Niaura R, Liu J, Lester BM. Smoking during pregnancy and newborn neurobehavior. Pediatrics 2003; 111: 1318-23.

64. Schoendorf KC, Kiely JL. Relationship of sudden infant death syndrome to maternal smoking during and after pregnancy. Pediatrics 1992; 90: 905-8.

65. Scher MS, Richardson GA, Robles N, Geva D, Goldschmidt L, Dahl RE, et al. Effects of prenatal substance exposure: altered maturation of Visual Evoked Potentials. Pediatr Neurol 1998; 18: 236-43.

66. Cornelius MD, Ryan CM, Day NL, Goldschmidt L, Willford JA. Prenatal tobacco effects on neuropsychological outcomes among preadolescents. J Dev Behav Pediatr 2001; 22: 217-25.

67. Williams GM, O'Callaghan M, Najman JM, Bor W, Andersen MJ, Richards D, et al. Maternal cigarette smoking and child psychiatric morbidity: a longitudinal study. Pediatrics 1998; 102(1): e11. Disponible en: http://pediatrics.aappublications.org/cgi/reprint/102/1/ e11 [consultado el 11/07/04].

68. Wakschlag LS, Lahey BB, Loeber R, Green SM, Gordon P.A, Leventhal BL. Maternal smoking during pregnancy and the risk of conduct disorder in boys. Arch Gen Psychiatry 1997; 54: 670-6.

69. Markussen Linnet K, Dalsgaard S, Obel C, Wisborg K, Henriksen TB, Rodriguez A, et al. Maternal lifestyle factors in pregnancy risk of Attention Deficit Hyperactivity Disorder and associated behaviors: review of the current evidence. Am J Psychiatry 2003; 160: 1028 - 40.

70. Muneoka K, Nakatsu T, Fuji JI, Ogawa T, Takigawa $M$. Prenatal administration of nicotine results in dopaminergic alterations in the neocortex. Neurotoxicol Teratol 1999; 21: 603-9.

71. Roy TS, Sabherwal U. Effects of gestational nicotine exposure on hippocampal morphology. Neurotoxicol Teratol 1998; 20: 465-73.

72. Navarro M, Rubio P, de Fonseca FR. Behavioural consequences of maternal exposure to natural cannabinoids in rats. Psychopharmacology (Berl) 1995; 122: 1-14.

73. Gotlib MS, Sassenrath EN, Chapman LF. Regulation of visual attention in offspring of female monkeys treated chronically with delta 9-tetrahydrocannabinol. Dev Psychobiol. 1981; 14: 507-12.

74. Fernandez-Ruiz JJ, Berrendero F, Hernandez ML, Romero J, Ramos JA. The endogenous cannabinoid system and brain development. Trends Neurosci 2000; 23: 14-20.

75. Wang X, Dow-Edwards D, Keller E, Hurd YL. Preferential limbic expression of the cannabinoid receptor mRNA in the human fetal brain. Neuroscience 2003; 118: 681-94.

76. Quiroga M. Cannabis: efectos nocivos sobre la salud física. Adicciones 2000; 12 Supl 2: 135-48.

77. Fried PA. Postnatal consequences of maternal marijuana use. In: Pinkert $T$, ed. Current research on the consequences of maternal drug abuse. NIDA Research Monograph N 59. Rockville, MD: National Institute on Drug Abuse, 1985. p 61-72.

78. Dahl RE, Scher MS, Williamson DE, Robles N, Day N. A longitudinal study of prenatal marijuana use. Effects on sleep and arousal at age 3 years. Arch Pediatrics Adolesc Med 1995; 149: 145-50.

79. Fried PA, Watkinson B, Gray R. Differential effects on cognitive functioning in 13- to 16-year-olds prenatally exposed to cigarettes and marihuana. Neurotoxicol Teratol 2003; 25: 427-36.

80. Fried PA, Smith AM. A literature review of the consequences of prenatal marihuana exposure. An emerging theme of a deficiency in aspects of executive function. Neurotoxicol Teratol 2001; 23: 1-11.

81. Koprich JB, Chen EY, Kanaan NM, Campbell NG, Kordower JH, Lipton JW. Prenatal 3,4-methylened ioxymethamphetamine (ecstasy) alters exploratory behaviour, reduces monoamine metabolism, and increases forebrain tyrosine hydroxylase fiber density of juvenile rats. Neurotoxicol Teratol 2003; 25: 50917.

82. Broening HW, Morford LL, Inman-Wood SL, Fukumura M, Vorhees CV. 3,4-methylenedioxymethamphe tamine (ecstasy)-induced learning and memory 
impairments depend on the age of exposure during early development. J Neurosci 2001; 21:3228-35.

83. Colado MI, O'Shea E, Granados R, Misra A, Murray TK, Green AR. A study of the neurotoxic effects of MDMA /"ecstasy") on 5-HT neurones in the brains of mothers and neonates following administration of the drug during pregnancy. Br J Pharmacol 1997; 124: 827-33.

84. Ho E, Karimi-Tabesh L, Koren G. Characteristics of pregnant women who use Ecstasy (3,4-methylenedio xymethamphetamine). Neurotoxicol Teratol 2001; 23: 561-7.

85. McElhatton PR. The effects of benzodiazepine use during pregnancy and lactation. Reprod Toxicol 1994; 8: 461-75.

86. Laegreid L, Hagberg G, Lundberg A. Neurodevelopment in late infancy after prenatal exposure to benzodiazepines: a prospective study. Neuropediatrics 1992; 23: 60-7.

87. Dolovitch LR, Addis A, Régis Vallancourt JM, Barry Power JD, Koren G, Einarson TR. Benzodiazepine use in pregnancy and major malformations or oral cleft: meta-analysis of cohort and case-control studies. BMJ 1998; 317 : 839-43.

88. González de Dios J, Moya-Benavetn M, CarrataláMarco F. Floppy infant en gemelos por uso de benzodiacepinas durante la gestación. Rev Neurol 1999; 29: 121-3.

89. Bracken MB, Triche EW, Belanger K, Hellenbrand K, Leaderer BP. Association of maternal caffeine consumption with decrements in fetal growth. Am J Epidemiol. 2003; 157: 456-66.

90. Jones $H E$, Balster RL. Inhalant abuse in pregnancy. Obstet Gynecol Clin North Am 1998; 25: 153-67.

91. Pajulo M, Savonlahti S, Piha P. Maternal substance abuse: infant psychiatric interest: a review and a hypothetical model of interaction. Am J Drug Alcohol Abuse 1999; 25: 761- 9.

92. Beeghly M, Frank DA; Rose-Jacobs $R$, Cabral $H$, Tronick E. Level of prenatal cocaine exposure and infant-caregiver attachment behavior. Neurotoxicol Teratol 2003; 25: 23-38.

93. O'Connor MJ, Kogan N, Findlay R. Prenatal alcohol exposure and attachment behavior in children. Alcohol Clin Exp Res 2002; 26: 1592-1602.

94. Miller BA, Smyth NJ, Mudar PJ. Mothers' alcohol and other drug problems and their punitiveness toward their children. J Stud Alcohol 1999; 60: 632-42.

95. Griffith DR, Freier C. Methodological issues in the assessment of the mother-child interactions of substance abusing women and their children. In: Kilbey MM, Asghar K (eds). Methodological issues in epidemiological, prevention, and treatment research on drug-exposed women and their children. NIDA
Research Monograph No 117. Rockville, MD: National Institutes on Drug Abuse, 1992, p 228-47.

96. Kettinger LA, Nair $P$, Schuler ME. Exposure to environmental risk factors and parenting attitudes among substance-abusing women. Am J Drug Alcohol Abuse 2000; 26: 1-11.

97. Lang AR, Pelham WE, Atkenson BM, Murphy DA. Effects of alcohol intoxication on parenting behavior in interactions with child confederates exhibiting normal or deviant behaviors. J Abnorm Child Psychol 1999; 27: 177-89.

98. Nicholson J, Biebel K, Hinden B, Henry A, Stier L. Critical issues for parents with mental illness and their families. Rockville. Center for Mental Health Services Research. Department of Psychiatry. University of Massachusetts Medical School; 2001.

99. Nicholson J, Sweeney EM, Geller JL. Focus on women: mothers with mental illness I. The competing demands of parenting and living with mental illness. Psychiatr Serv 1998; 49: 635-42.

100. Nicholson J, Sweeney EM, Geller JL. Focus on women: mothers with mental illness II. Family relationships and the context of parenting. Psychiatr Serv 1998; 49: 643-49.

101. Espinosa M, Beckwith L, Howard J, Tyler R, Swanson K. Maternal psychopathology and attachment in toddlers of heavy cocaine-using mothers. Infant Mental Health Journal 2001; 22: 316-33.

102. Weissman MM, McAvay G, Goldstein RB, Nunes E, Verdeli H, Wickramaratne P. Risk/protective factors among addicted mothers' offspring: a replication study. Am J Drug Alcohol Abuse 1999; 25: 661-79.

103. Najavits LM, Weiss RD, Shaw SR. The link between substance abuse and posttraumatic stress disorder in women: a research review. Am J Addict 1997; 6: 27383.

104. Stocco P. Nuevas perspectivas en la prevención e intervención sobre la mujer toxicómana en Europa. Ponencia presentada al I Symposium Nacional sobre Adicción en la Mujer. Fundación Instituto Spiral. Madrid, 6-7 de Octubre de 2000. Disponible en: http://www. institutospiral.com/cursos\%20y\%20seminarios/ primersymposium [consultado el 11/07/04].

105. Martin SL, Beaumont JL, Kupper LL. Substance use before and during pregnancy: links to intimate partner violence. Am J Drug Alcohol Abuse 2003; 29: 599617.

106. Kauffmann J, Plotsky PM, Nemeroff CB, Charney D. Effects of early adverse experiences on brain structure and function: clinical implications. Biol Psychiatry 2000; 48: 778-90.

107. Spear LP, Campbell J, Snyder K, Silveri A, Katovic N. Animal behavior models. Increased sensitivity to stressors and other environmental experiences after 
prenatal cocaine exposure. Ann N Y Acad Sci 1998; 846: 76-88.

108. Woods JR. Clinical management of drug dependency in pregnancy. In: Chiang CN, Finnegan LP, eds. Medications development for the treatment of pregnant addicts and their infants. NIDA Research Monograph No 149. Rockville, MD: National Institutes on Drug Abuse, 1995. p 39-57.

109.Fischer G, Schmidl-Mohl K, Etzersdorfer P, Schatten C, Langer M, Weniger $M$, et al. Approaches to the management of pregnant drug misusers. In Pompidou Group (ed). Pregnancy and drug misuse: Proceedings. Symposium. Strasbourg, 6-7 March 1997. Strasbourg: Council of Europe Publishing, 1998. p 15-26.

110. Kumpfer KL. Links between prevention and treatment for drug-abusing women and their children. In: Wetherington $C L$, Roman $A B$, eds. Drug addiction research and the health of women. Rockville, MD: National Institute on Drug Abuse, 1998, p 417-38.

111. LLopis JJ. Sexuality and addiction. Implication in the woman drug addict. In: Stocco P, LLopis JJ, DeFazio L, Calafat A, Mendes F. Women drug abuse in Europe: gender identity. Venezia: IREFREA. 2000, p 39-44.

112. Seiden AM. Measures of pregnant, drug-abusing women for treatment research. In: Kilbey MM, Ashgar $\mathrm{K}$, eds. Methodological issues in epidemiological, prevention, and treatment research on drug-exposed women and their children. NIDA Research Monograph $N^{\circ}$ 117. Rockville, MD: National Institutes on Drug Abuse, 1992. p 194-211.

113. Klee $\mathrm{H}$. Health care delivery to drug using women during pregnancy and early motherhood. In Pompidou Group, ed. Pregnancy and drug misuse: Proceedings. Symposium. Strasbourg, 6-7 March 1997. Strasbourg: Council of Europe Publishing, 1998. p.27-41.

114. Llopis JJ, Rebollida M. Clínica de la mujer toxicómana. Un análisis de actitudes. Ponencia presentada al I Symposium Nacional sobre Adicción en la Mujer. Fundación Instituto Spiral. Madrid, 6-7 de Octubre de 2000. Disponible en: http://www.institutospiral. com/cursos $\% 20 y \% 20$ seminarios/primersymposium [consultado el 11/07/04].

115. Rosenbaum M, Irwin K. Pregnancy, drugs, and harm reduction. In: Wetherington $C L$, Roman $A B$, eds. Drug addiction research and the health of women. Rockville, MD: National Institute on Drug Abuse, 1998. p 30918.

116. Klee $\mathrm{H}$. Health care delivery during pregnancy: health professionals' views. In Pompidou Group, ed. Pregnancy and drug misuse: Proceedings. Symposium organised by the Co-operation Group to Combat Drug Abuse and Illicit Trafficking in Drugs (Pompidou Group). Strasbourg, 6-7 March 1997. Strasbourg: Council of Europe Publishing, 1998. p 23-54.

117. Vulnerable people. Addicted mothers and their young children. Project supported by the European Comission. Bélgica 2001. Disponible en: http://www. vulnerablepeople.org/opmaak/manual.pdf [consultado el 11/07/04]

118. Paltrow LM. Punishing women for their behaviour during pregnancy: an approach that undermines the health of women and children. In: Wetherington $\mathrm{CL}$, Roman $A B$ (eds). Drug addiction research and the health of women. Rockville, MD: National Institute on Drug Abuse, 1998, p 467-501.

119. Howard J, Beckwith L. Issues in subject recruitment and retention with pregnant and parenting substanceabusing women. In: Rahdert ER, ed. Treatment for drug-exposed women and their children: advances in research methodology. NIDA Research Monograph $\mathrm{N}^{\circ}$ 166. Rockville, MD: National Institute on Drug Abuse, 1996. p 68-86.

120.Daley D, Gorske T. Improving treatment adherence for mothers with substance abuse problems. The Source. Newsletter of the National Abandoned Infants Assistance Resource Center 2000; 10: 1-5.

121. Becker J, Duffy C. Women drug users and drugs services provision: service-levels responses to engagement and retention. DPAS Paper 17. London: Report for the Home Office Drugs Safety Strategy Directorate, 2002.

122. Laudet A, Magura S, Furst RT, Kumar N, Whitney S. Male partners of substance-abusing women in treatment: an exploratory study. Am J Drug Alcohol Abuse 1999; 25: 607-27.

123. Sirvent C. Las dependencias relacionales (D.R.): dependencia emocional, codependencia y bidependencia. Ponencia presentada al I Symposium Nacional sobre Adicción en la Mujer. Fundación Instituto Spiral. Madrid, 6-7 de Octubre de 2000. Disponible en: http://www.institutospiral.com/ cursos $\% 20$ y 20 seminarios/primersymposium [consultado el 11/07/04].

124. Falkin GP, Strauss SM. Social supporters and drug use enablers: a dilemma for women in recovery. Addict Behav 2003; 28: 141-55.

125. Pinazi S, Ferrero C. Impacto psicosocial del acogimiento familiar en familia extensa: el caso de las abuelas y los abuelos acogedores. Rev Mult Geront 2003; 13: 89101. 